\title{
Gradient flows in the normal and Kähler metrics and triple bracket generated metriplectic systems
}

\author{
Anthony M. Bloch, Philip J. Morrison, and Tudor S. Ratiu
}

\begin{abstract}
The dynamics of gradient and Hamiltonian flows with particular application to flows on adjoint orbits of a Lie group and the extension of this setting to flows on a loop group are discussed. Different types of gradient flows that arise from different metrics including the so-called normal metric on adjoint orbits of a Lie group and the Kähler metric are compared. It is discussed how a Kähler metric can arise from a complex structure induced by the Hilbert transform. Hybrid and metriplectic flows that arise when one has both Hamiltonian and gradient components are examined. A class of metriplectic systems that is generated by completely antisymmetric triple brackets is described and for finitedimensional systems given a Lie algebraic interpretation. A variety of explicit examples of the several types of flows are given.
\end{abstract}

Keywords: loop groups, adjoint orbits, Hamiltonian systems, integrable systems, gradient flows, metriplectic systems, thermodynamics

\section{Introduction}

Dynamical systems, finite or infinite, that describe physical phenomena typically have parts that are in some sense Hamiltonian and parts that can be recognized as dissipative, with the Hamiltonian part being generated by a Poisson bracket and the dissipative part being some kind of gradient flow. The description of Hamiltonian systems has received much attention over nearly two centuries and, although some forms of dissipation have received general attention, the understanding and classification of dissipative dynamics is a much broader topic and consequently less well developed. Early modern treatments of geometric Hamiltonian mechanics include those of Souriau [1970] and Abraham and Marsden [1978], and the literature on this topic is now immense. A special type of gradient flow that preserves invariants, the double bracket formalism described in Brockett [1991] (see, e.g., Bloch [1990], Bloch [2003]), is a formalism that occurs in a variety of contexts (see Bloch, Krishnaprasad, Marsden, and Ratiu [1994, 1996]) and is welladapted to practical numerical computations (see Vallis, Carnevale and Young [1989]; Flierl and Morrison [2011]). Examples of infinite-dimensional gradient flows include the Cahn-Hilliard systems (see Otto [2001]) and the celebrated Ricci flows (see Hamilton [1982]; Chow [2004]), which are nonlinear diffusion-like equations. A general form for combined Hamiltonian and gradient flows was described in Morrison [1986], where such flows were termed metriplectic flows (see also Oettinger [2006]; Morrison [2009]; Liero and Mielke [2012]). Thus, it is evident that there are a variety Hamiltonian and dissipative flows, and the purpose of this paper is to explore the form and geometric structure of such flows in both the ode and pde contexts.

Anthony M. Bloch

Department of Mathematics, The University of Michigan, 530 Church Street, Ann Arbor, MI 48109-1043, USA. abloch@umich . edu

Philip J. Morrison

Department of Physics and Institute for Fusion Studies, University of Texas, 2515 Speedway Stop C1600, Austin, TX 78712-0264, USA.

morrison@physics.utexas.edu

Tudor S. Ratiu

Department of Mathematics and Bernoulli Center, Ecole Polytechnique Fédérale de Lausanne, CH-1015 Lausanne, Switzerland. tudor.ratiudepfl.ch 
Specifically, in this paper we discuss the dynamics of gradient and Hamiltonian flows, with particular application to flows on adjoint orbits of a Lie group and the extension of this setting to flows on a loop group. We compare the different types of gradient flows that arise from different metrics, in particular, the so-called normal metric on adjoint orbits of a Lie group and the Kähler metric. We discuss how a Kähler metric can arise from the complex structure induced from the Hilbert transform. We also consider flows that arise when one has both Hamiltonian and gradient structures present. In particular, we discuss metriplectic flows, flows that produce entropy while conserving energy. We consider such flows in both the finite and infinite settings, and discuss a general class of metriplectic flows that arise from completely antisymmetric triple brackets. For finite systems, we show how the triple bracket has a natural Lie algebraic formulation, and for infinite systems we give a procedure for constructing a quite general class of metriplectic pdes. We also consider, hybrid flows, of Hamiltonian and gradient form, that dissipate energy. Several examples of hybrid and metriplectic flows are given, including finite systems such as the Toda lattice on $\mathbb{R}$ and metriplectic $\mathfrak{s o}(3)$ brackets. Various infinite-dimensional examples including a $1+1$ dissipative systems that conserves energy, and hybrid systems such as the KdV with dissipation, the Ott and Sudan [1969] equation that describes Landau damping, and others.

The paper is organized as follows. In section 2 we review material need for latter development. In particular, we discuss metrics on adjoint orbits, Toda flows and the double bracket formulation. Sections 3 and 4 contain the main new results of the paper as described above. In section 3 we discuss metrics on loop groups and related gradient flows, while in section 4 we discuss our results on metriplectic systems, in both finite- and infinite-dimensions, and give examples.

\section{Metrics on adjoint orbits of compact Lie groups and associated dynamical systems}

\subsection{Double bracket systems}

Let $\mathfrak{g}_{u}$ be the compact real form of a complex semisimple Lie algebra $\mathfrak{g}, G_{u}$ a compact connected real Lie group with Lie algebra $\mathfrak{g}_{u}$, and $\kappa$ the Killing form (on $\mathfrak{g}$ or $\mathfrak{g}_{u}$, depending on the context).

The "normal" metric on the adjoint orbit $\mathscr{O}$ of $G_{u}$ through $L_{0} \in \mathfrak{g}_{u}$ (see Atiyah [1982], [Besse, 2008, Chapter 8]) is given as follows. Decompose orthogonally $\mathfrak{g}_{u}=\mathfrak{g}_{u}^{L} \oplus \mathfrak{g}_{u L}$, relative to to the invariant inner product $\langle\rangle:,=-\kappa(, \quad)$, where $\mathfrak{g}_{u L}:=\operatorname{kerad}_{L}$ is the centralizer of $L$ and $\mathfrak{g}_{u}^{L}=\operatorname{rangead}_{L}$; as usual, ad $\operatorname{ad}_{L}:=[L, \cdot]$. For $X \in \mathfrak{g}_{u}$ denote by $X^{L} \in \mathfrak{g}_{u}^{L}$ and $X_{L} \in \mathfrak{g}_{u L}$ the orthogonal projections of $X$ on $\mathfrak{g}_{u}^{L}$ and $\mathfrak{g}_{u L}$, respectively. Recall that a general vector tangent at $L$ to the adjoint orbit $\mathscr{O}$ is necessarily of the form $[L, X]$ for some $X \in \mathfrak{g}_{u}$. The normal metric on $\mathscr{O}$ is the $G_{u}$-invariant Riemannian metric given by

$$
\langle[L, X],[L, Y]\rangle_{\text {normal }}:=\left\langle X^{L}, Y^{L}\right\rangle
$$

for any $X, Y \in \mathfrak{g}_{u}$.

Fix $N \in \mathfrak{g}_{u}$ and consider the flow on the adjoint orbit $\mathscr{O}$ of $G_{u}$ through $L_{0} \in \mathfrak{g}_{u}$ given by

$$
\frac{d}{d t} L(t)=[L(t),[L(t), N]], \quad L(0)=L_{0} \in \mathfrak{g}_{u} .
$$

We recall the following well-known result (Brockett [1991], Brockett [1994], Bloch. Brockett, and Ratiu [1990], Bloch. Brockett, and Ratiu [1992], Bloch. Flaschka, and Ratiu [1990], Bloch and Iserles [2005]).

Proposition 1. The vector field given by the ordinary differential equation (2) is the gradient of the function $H(L)=$ $\kappa(L, N)$ relative to the normal metric on $\mathscr{O}$.

Proof. By the definition of the gradient $\operatorname{grad} H(L) \in T_{L} \mathscr{O} \subset \mathfrak{g}_{u}$ relative to the normal metric, we have for any $L \in \mathscr{O}$ and $\delta L \in \mathfrak{g}_{u}$,

$$
d H(L) \cdot[L, \delta L]=\langle\operatorname{grad} H(L),[L, \delta L]\rangle_{\text {normal }}
$$

where $\cdot$ denotes the natural pairing between 1 -forms and tangent vectors and $[L, \delta L]$ is an arbitrary tangent vector at $L$ to $\mathscr{O}$. Set $\operatorname{grad} H(L)=[L, X]=\left[L, X^{L}\right]$. Then (3) becomes

$$
-\langle[L, \delta L], N\rangle=\langle[L, X],[L, \delta L]\rangle_{\text {normal }}
$$

or, equivalently, 


$$
\langle[L, N], \delta L\rangle=\left\langle X^{L}, \delta L^{L}\right\rangle=\left\langle X^{L}, \delta L\right\rangle .
$$

Since $[L, N] \in \mathfrak{g}_{u}^{L}$, this implies that $X^{L}=[L, N]$, and hence $\operatorname{grad} H(L)=[L,[L, N]]$, as stated.

The same computation, for a general function $H \in C^{\infty}\left(\mathfrak{g}_{u}\right)$, yields

$$
\operatorname{grad} H(L)=-[L,[L, \nabla H(L)]]
$$

where $\nabla H(L)$ denotes the gradient of the function $H$ relative to the invariant inner product $\langle\rangle:,=-\kappa($, $)$, i.e., $d H(L) \cdot X=\langle\nabla H(L), X\rangle$ for any $X \in \mathfrak{g}_{u}$.

\subsection{The finite Toda system}

The double bracket equation (2) is intimately related to the finite non-compact Toda lattice system. This is a Hamiltonian system modeling $n$ particles moving freely on the $x$-axis and interacting under an exponential potential. Denoting the position of the $k$ th particle by $x_{k}$, the Hamiltonian is given by

$$
H(x, y)=\frac{1}{2} \sum_{k=1}^{n} y_{k}^{2}+\sum_{k=1}^{n-1} e^{x_{k}-x_{k+1}}
$$

and hence the associated Hamiltonian equations are

$$
\dot{x}_{k}=\frac{\partial H}{\partial y_{k}}=y_{k}, \quad \dot{y}_{k}=-\frac{\partial H}{\partial x_{k}}=e^{x_{k-1}-x_{k}}-e^{x_{k}-x_{k+1}},
$$

where we use the conventions $e^{x_{0}-x_{1}}=e^{x_{n}-x_{n+1}}=0$, which corresponds to formally setting $x_{0}=-\infty$ and $x_{n+1}=+\infty$.

This system of equations has an extraordinarily rich structure. Part of this is revealed by Flaschka's change of variables (Flaschka [1974]) given by

$$
a_{k}=\frac{1}{2} e^{\left(x_{k}-x_{k+1}\right) / 2} \quad \text { and } \quad b_{k}=-\frac{1}{2} y_{k} .
$$

which transform (5) to

$$
\left\{\begin{array}{l}
\dot{a}_{k}=a_{k}\left(b_{k+1}-b_{k}\right), \quad k=1, \ldots, n-1, \\
\dot{b}_{k}=2\left(a_{k}^{2}-a_{k-1}^{2}\right), \quad k=1, \ldots, n,
\end{array}\right.
$$

with the boundary conditions $a_{0}=a_{n}=0$. This system is equivalent to the Lax equation

$$
\frac{d}{d t} L=[B, L]=B L-L B,
$$

where

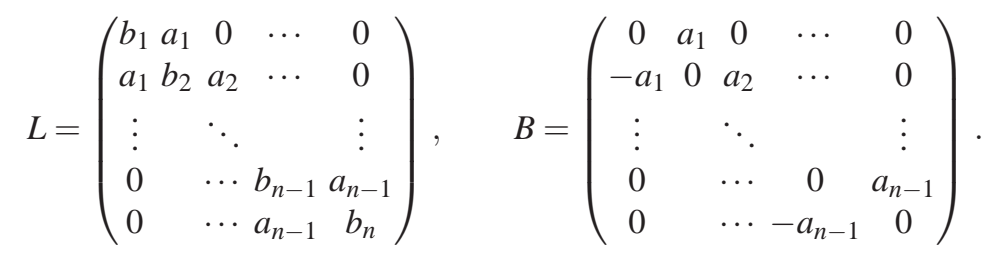

If $O(t)$ is the orthogonal matrix solving the equation

$$
\frac{d}{d t} O=B O, \quad O(0)=\text { Identity },
$$

then from (7) we have

$$
\frac{d}{d t}\left(O^{-1} L O\right)=0 .
$$


Thus, $O^{-1} L O=L(0)$, i.e., $L(t)$ is related to $L(0)$ by conjugation with an orthogonal matrix and thus the eigenvalues of $L$, which are real and distinct, are preserved along the flow. This is enough to show that this system is explicitly solvable or integrable. Equivalently, after fixing the center of mass, i.e., setting $b_{1}+\cdots+b_{n}=0$, the $n-1$ integrals in involution whose differentials are linearly independent on an open dense set of phase space $\left\{\left(a_{1}, \ldots, a_{n-1}, b_{1}, \ldots, b_{n}\right) \mid\right.$ $\left.b_{1}+\cdots+b_{n}=0\right\}$ are $\operatorname{Tr} L^{2}, \ldots, \operatorname{Tr} L^{n}$.

\subsection{Lie algebra integrability of the Toda system}

Let us quickly recall the well-known Lie algebraic approach to integrability of the Toda lattice. Let $\mathfrak{g}$ be a Lie algebra with an invariant non-degenerate bilinear symmetric form $\langle$,$\rangle , i.e., \langle[\xi, \eta], \zeta\rangle=\langle\xi,[\eta, \zeta]\rangle$ for all $\xi, \eta, \zeta \in \mathfrak{g}$ and $\langle\xi, \cdot\rangle=0$ implies $\xi=0$. Suppose that $\mathfrak{k}, \mathfrak{s} \subset \mathfrak{g}$ are Lie subalgebras and that, as vector spaces, $\mathfrak{g}=\mathfrak{k} \oplus \mathfrak{s}$. Let $\pi_{\mathfrak{k}}: \mathfrak{g} \rightarrow \mathfrak{k}$, $\pi_{\mathfrak{s}}: \mathfrak{g} \rightarrow \mathfrak{s}$ be the two projections induced by this vector space direct sum decomposition. Since $\mathfrak{g} \ni \xi \stackrel{\sim}{\longmapsto}\langle\xi, \cdot\rangle \in \mathfrak{g}^{*}$ is a vector space isomorphism, it naturally induces the isomorphisms $\mathfrak{k}^{\perp} \cong \mathfrak{s}^{*}, \mathfrak{s}^{\perp} \cong \mathfrak{k}^{*}$. By non-degeneracy of $\langle$,$\rangle , we$ have $\mathfrak{g}=\mathfrak{s}^{\perp} \oplus \mathfrak{k}^{\perp}$; denote by $\pi_{\mathfrak{k} \perp}: \mathfrak{g} \rightarrow \mathfrak{k}^{\perp}, \pi_{\mathfrak{s}^{\perp}}: \mathfrak{g} \rightarrow \mathfrak{s}^{\perp}$ the two projections induced by this vector space direct sum decomposition. In particular, $\mathfrak{g}, \mathfrak{s}^{\perp}, \mathfrak{k}^{\perp}$ all carry natural Lie-Poisson structures. The (-)Lie-Poisson bracket of $\mathfrak{s}^{*} \cong \mathfrak{k}^{\perp}$ is given by

$$
\{\varphi, \psi\}(\xi)=-\left\langle\xi,\left[\pi_{\mathfrak{s}} \nabla \varphi(\xi), \pi_{\mathfrak{s}} \nabla \psi(\xi)\right]\right\rangle, \quad \xi \in \mathfrak{k}^{\perp},
$$

where $\varphi, \psi: \mathfrak{k}^{\perp} \rightarrow \mathbb{R}$ are any smooth functions, extended arbitrarily to smooth functions, also denoted by $\varphi$ and $\psi$, on $\mathfrak{g}$ and $\nabla \varphi, \nabla \psi$ are the gradients of these arbitrary extensions relative to $\langle$,$\rangle . This formula follows from the fact that the$ gradient on $\mathfrak{k}^{\perp}$ of $\left.\varphi\right|_{\mathfrak{k} \perp}$, which is an element of $\mathfrak{s}$ due to the isomorphism $\mathfrak{k}^{\perp} \cong \mathfrak{s}^{*}$, equals $\pi_{\mathfrak{s}} \nabla \varphi$. Thus, the Hamiltonian vector field of $\psi \in C^{\infty}\left(\mathfrak{k}^{\perp}\right)$, given by $\dot{\varphi}=\{\varphi, \psi\}$ for any $\varphi \in C^{\infty}\left(\mathfrak{k}^{\perp}\right)$, has the expression

$$
X_{\psi}(\xi)=-\pi_{\mathfrak{k}^{\perp}}\left[\pi_{\mathfrak{s}} \nabla \psi(\xi), \xi\right], \quad \xi \in \mathfrak{k}^{\perp}
$$

with the same conventions as above.

If $\psi \in C^{\infty}(\mathfrak{g})$ is invariant, i.e., $[\nabla \psi(\zeta), \zeta]=0$ for all $\zeta \in \mathfrak{g}$, then (10) simplifies to

$$
X_{\psi}(\xi)=\left[\pi_{\mathfrak{k}} \nabla \psi(\xi), \xi\right]=-\left[\pi_{\mathfrak{s}} \nabla \psi(\xi), \xi\right], \quad \xi \in \mathfrak{k}^{\perp} .
$$

The Adler-Kostant-Symes Theorem (see Adler [1979], Kostant [1979], Symes [1980a b], and Ratiu [1980] for many theorems of the same type) states that if $\varphi$ and $\psi$ are both invariant functions on $\mathfrak{g}$, then $\{\varphi, \psi\}=0$ on $\mathfrak{k}^{\perp}$ which is equivalent to the commutation of the flows of the Hamiltonian vector fields 111 .

Suppose that $G=K S$, where $G$ is a Lie group with Lie algebra $\mathfrak{g}$ and $K, S \subset G$ are closed subgroups with Lie algebras $\mathfrak{k}$ and $\mathfrak{s}$, respectively. The writing $G=K S$ means that each element $g \in G$ can be uniquely decomposed as $g=k s$, where $k \in K$ and $s \in S$ and that this decomposition defines a smooth diffeomorphism $K \times S \approx G$. The coadjoint action of $S$ on $\mathfrak{s}^{*}$ has the following expression, if $\mathfrak{s}^{*}$ is identified with $\mathfrak{k}^{\perp}$ via $\langle\rangle:$, if $s \in S, \xi \in \mathfrak{k}^{\perp}$, then $s \cdot \xi=\pi_{\mathfrak{k} \perp} \operatorname{Ad}_{s} \xi$, where $\operatorname{Ad}_{s} \xi$ is the adjoint action in $G$ of the element $s \in S \subset G$ on $\xi \in \mathfrak{k}^{\perp} \subset \mathfrak{g}$.

For the Toda lattice (7), this general setup applies in the following way. Let $G=\mathrm{GL}(n, \mathbb{R}), K=\mathrm{SO}(n), S=$ \{invertible lower triangular matrices $\}, G=K S$ is the Gram-Schmidt orthonormalization process, $\mathfrak{g}=\mathfrak{g l}(n, \mathbb{R}), \mathfrak{k}=$ $\mathfrak{s o}(n), \mathfrak{s}=\{$ lower triangular matrices $\},\langle\xi, \eta\rangle:=\operatorname{Tr}(\xi \eta)$ for all $\xi, \eta \in \mathfrak{g l}(n, \mathbb{R}), \mathfrak{k}^{\perp}=\mathfrak{s y \mathfrak { m }}(n)$ the vector space of symmetric matrices, and $\mathfrak{s}^{\perp}=\mathfrak{n}$, the nilpotent Lie algebra of strictly lower triangular matrices. The set of matrices $L$ in (8) is a union of $S$-coadjoint orbits parametrized by the value of the trace; for example, the set of trace zero matrices $L$ of the form (8) equals the $S$-coadjoint orbit through the symmetric matrix that has everywhere zero entries with the exception of the upper and lower first diagonals where all entries are equal to one. Thus, the Toda lattice is a Poisson system whose restriction to a symplectic leaf is a classical Hamiltonian system with $n-1$ degrees of freedom. The Hamiltonian of the Toda lattice is $\frac{1}{2} \operatorname{Tr} L^{2}$ and the $f_{k}(L):=\frac{1}{k} \operatorname{Tr} L^{k}, k=1, \ldots, n-1$ are the $n-1$ integrals in involution (by the Adler-Kostant-Symes Theorem) and are generically independent. 


\subsection{The Toda system as a double bracket equation}

If $N$ is the matrix $\operatorname{diag}\{1,2, \ldots, n\}$, the Toda equations (7) may be written in the double bracket form (2) for $B:=[N, L]$. This was shown in Bloch [1990]; the consequences of this fact were further analyzed for general compact Lie algebras in Bloch, Brockett, and Ratiu [1990], Bloch, Brockett, and Ratiu [1992], and Bloch, Flaschka, and Ratiu [1990]. As shown in Proposition 1 the double bracket equation, with $L$ replaced by $i L$ and $N$ by i $N$, restricted to a level set of the integrals described above, i.e., restricted to a generic adjoint orbit of $\mathrm{SU}(n)$, is the gradient flow of the function $\operatorname{Tr} L N$ with respect to the normal metric; see Bloch. Flaschka, and Ratiu [1990] for this approach.

This observation easily implies that the flow tends asymptotically to a diagonal matrix with the eigenvalues of $L(0)$ on the diagonal and ordered according to magnitude, recovering the result of Moser [1975], Symes [1982], and Deift, Nanda, and Tomei [1983].

\subsection{Riemannian metrics on $\mathscr{O}$}

Now, we recall that, in addition to the normal metric on an adjoint orbit, there are other natural $G_{u}$-invariant metrics: the induced and the group invariant Kähler metrics (as discussed in [Atiyah, 1982, §4], Atiyah and Pressley [1983], and [Besse, 2008, Chapter 8]).

Firstly, there is the induced metric $b$ on $\mathscr{O}$, defined by $b:=\imath^{*}(-\kappa()$,$) , where \imath: \mathscr{O} \hookrightarrow \mathfrak{g}_{u}$ is the inclusion and $\langle\rangle:,=-\kappa(, \quad)$ is thought of as a constant Riemannian metric on $\mathfrak{g}_{u}$. Therefore,

$$
b(L)([L, X],[L, Y]):=\langle[L, X],[L, Y]\rangle
$$

for any $L \in \mathscr{O}, X, Y \in \mathfrak{g}_{u}$. The induced metric on $\mathscr{O}$ is also $G_{u}$-invariant.

Secondly, there are the $G_{u}$-invariant Kähler metrics on $\mathscr{O}$ compatible with the natural complex structure (of course, induced by the complex structure of $G$ ). These are in bijective correspondence (by the transgression homomorphism) with the set of $G_{u}$-invariant sections of the trivial vector bundle over $\mathscr{O}$ whose fiber at $L \in \mathscr{O}$ is the center of $\operatorname{ker}\left(\operatorname{ad}_{L}\right)$ and whose scalar product with all positive roots is positive ([Besse, 2008, Proposition 8.83]). Among these, there is the $G_{u}$-invariant Kähler metric $b_{2}$ which is compatible with both the natural complex structure on $\mathscr{O}$ and has as imaginary part the orbit symplectic structure; $b_{2}$ is called the standard Kähler metric on $\mathscr{O}$.

The $G_{u}$-invariant Riemannian metrics on a maximal dimensional orbit $\mathscr{O}$ are completely determined by $T$-invariant inner products on the direct sum of the two dimensional root spaces of $\mathfrak{g}_{u}$, which is the tangent space to $\mathscr{O}$ at the point $L_{0} \in \mathfrak{t}$ in the interior of the positive Weyl chamber; recall that $\mathscr{O}$ intersects the positive Weyl chamber in a unique point. The negative of the Killing form induces on each such 2-dimensional space an inner product. This inner product, left translated at all points of $\mathscr{O}$ by elements of $G_{u}$, yields the normal metric on $\mathscr{O}$. Any other $G_{u}$-invariant inner product on $\mathscr{O}$ is obtained by left translating at all points of $\mathscr{O}$ the inner product on this direct sum of 2 -dimensional root spaces obtained by multiplying in each 2-dimensional summand the inner product with a positive real constant.

Since $L_{0}$ lies in the interior of the positive Weyl chamber (because $\mathscr{O}$ is maximal dimensional), $\alpha\left(L_{0}\right)>0$ for all positive roots $\alpha$ of $\mathfrak{g}_{u}$. Then the constant by which the natural inner product on the 2-dimensional root space needs to be multiplied in order to get the standard Kähler metric is $\alpha\left(L_{0}\right)$, whereas to get the induced metric, it is $\alpha\left(L_{0}\right)^{2}$ ([Atiyah, 1982, Remark 2 in §4]). We can formulate this differently, as in Bloch, Flaschka, and Ratiu [1990]. Since, by (12) and (1),

$$
\begin{aligned}
b(L)([L, X],[L, Y]) & =\langle[L, X],[L, Y]\rangle=\left\langle\left[L, X^{L}\right],\left[L, Y^{L}\right]\right\rangle=\left\langle-\left[L,\left[L, X^{L}\right]\right], Y^{L}\right\rangle=\left\langle-\left[L,\left[L, X^{L}\right]\right]^{L}, Y^{L}\right\rangle \\
& =\left\langle-\operatorname{ad}_{L}^{2}[L, X],[L, Y]\right\rangle_{\text {normal }}
\end{aligned}
$$

we have

$$
b(L)([L, X],[L, Y])=b_{1}(L)\left(\mathscr{A}(L)^{2}[L, X],[L, Y]\right)
$$

where we denote now by $b_{1}$ the normal metric and $\mathscr{A}(L):=\sqrt{\left(\operatorname{iad}_{L}\right)^{2}}$ is the positive square root of $\left(\operatorname{iad}_{L}\right)^{2}=-\operatorname{ad}_{L}^{2}=$ $\mathscr{A}(L)^{2}$. The standard Kähler metric on $\mathscr{O}$ is then given by

$$
\left.b_{2}(L)[L, X],[L, Y]\right)=b_{1}(\mathscr{A}(L)[L, X],[L, Y]) \text {. }
$$


Note that, as opposed to the normal and induced metrics which have explicit expressions, the standard Kähler metric on $\mathscr{O}$ requires the spectral decomposition of $\mathscr{A}(L)$ at any point $L \in \mathscr{O}$. Or, as explained above, one expresses it at the point $L_{0}$ in the positive Weyl chamber in terms of the positive roots and then left translates the resulting inner product at any point of $\mathscr{O}$. The normal metric does not depend on the operators $\mathscr{A}(L)$, whereas the standard Kähler and induced metrics do.

\section{Gradient flows on the loop group of the circle}

In this section we introduce three weak Riemannian metrics on the subgroup of average zero functions of the connected component of the loop group $\widetilde{\mathrm{L}}\left(S^{1}\right)$ of the circle, analogous to the normal, standard Kähler, and induced metrics on adjoint orbits of compact semisimple Lie groups. Of course, we shall not work on adjoint orbits of this group because they degenerate to points, $\widetilde{\mathrm{L}}\left(S^{1}\right)$ being a commutative group. Then we shall compute the gradient flows for these three metrics.

\subsection{The loop group of $S^{1}$}

Recall (e.g., Pressley and Segal [1986) that the loop group $\widetilde{\mathrm{L}}\left(S^{1}\right)$ of the circle $S^{1}$ consists of smooth maps of $S^{1}$ to $S^{1}$. With pointwise multiplication, $\widetilde{\mathrm{L}}\left(S^{1}\right)$ is a commutative group. Often, elements of $\widetilde{\mathrm{L}}\left(S^{1}\right)$ are written as $e^{\mathrm{i} f}$, where $f \in \widetilde{\mathrm{L}}(\mathbb{R}):=\left\{g:[-\pi, \pi] \rightarrow \mathbb{R} \mid g\right.$ is $C^{\infty}, g(\pi)=g(-\pi)+2 n \pi$, for some $\left.n \in \mathbb{Z}\right\} ; n$ is the winding number of the closed curve $[-\pi, \pi] \ni t \mapsto e^{\mathrm{i} g(t)} \in S^{1}$ about the origin. More precisely, there is an exact sequence of groups

$$
\begin{aligned}
0 \longrightarrow \mathbb{Z} & \longrightarrow \widetilde{\mathrm{L}}(\mathbb{R}) \stackrel{\widetilde{\exp }}{\longrightarrow} \widetilde{\mathrm{L}}\left(S^{1}\right) \longrightarrow \underset{\mathbb{Z}}{\longrightarrow} \longrightarrow 0 \\
n & \longmapsto 2 \pi n ; \quad f \longmapsto e^{\mathrm{i} f} \longmapsto \frac{f(\pi)-f(-\pi)}{2 \pi}
\end{aligned}
$$

which shows that kerexp $=\mathbb{Z}$ and cokerexp $=\{0\}$. Thus the connected components of $\widetilde{\mathrm{L}}\left(S^{1}\right)$ are indexed by the winding number. The connected component of the identity $\widetilde{\mathrm{L}}\left(S^{1}\right)_{0}$ consists of loops with winding number zero about the origin.

If one insists on working with smooth loops, then one can consider $\widetilde{\mathrm{L}}\left(S^{1}\right)$ and $\widetilde{\mathrm{L}}\left(S^{1}\right)_{0}$ as Fréchet Lie groups either in the convenient calculus of Kriegl and Michor [1997] or in the tame category of Hamilton [1982].

Alternatively, one can work with loops $e^{\mathrm{i} f}$ for $f:[-\pi, \pi] \rightarrow \mathbb{R}$ of Sobolev class $H^{s}$, where $s \geq 1$ (or appropriate $W^{s, p}$ or Hölder spaces). By standard theory (see, e.g., Palais [1968] or Adams and Fournier [2003]), it is checked that $\widetilde{\mathrm{L}}\left(S^{1}\right)$ is a Hilbert Lie group (see, e.g., Bourbaki [1971] or Neeb [2004]). We shall not add the index $s$ on $\widetilde{\mathrm{L}}(\mathbb{R})$ and $\widetilde{\mathrm{L}}\left(S^{1}\right)$; from now on we work exclusively in this category of $H^{s}$ Sobolev class maps and loops. A simple proof of the fact that $\widetilde{L}(\mathbb{R})$ is a Hilbert Lie group was given to us by K.-H. Neeb. First, note that $\widetilde{L}(\mathbb{R})$ is a closed additive subgroup of the Hilbert space $H^{s}(\mathbb{R}):=\left\{h: \mathbb{R} \rightarrow \mathbb{R} \mid h\right.$ of class $\left.H^{s}\right\}$. Second, $\widetilde{\mathrm{L}}(\mathbb{R})=\widetilde{\mathrm{L}}(\mathbb{R})_{0} \times \mathbb{Z}$ as topological groups, where $\widetilde{\mathrm{L}}(\mathbb{R})_{0}:=\{g \in \widetilde{\mathrm{L}}(\mathbb{R}) \mid g(\pi)=g(-\pi)\}$ is the closed vector subspace of $H^{s}(\mathbb{R})$ consisting of periodic functions; hence it is an additive Hilbert Lie group. Therefore, there is a unique Hilbert Lie group structure on $\widetilde{L}(\mathbb{R})$ for which $\widetilde{\mathrm{L}}(\mathbb{R})_{0}$ is the connected component of the identity. For general criteria that characterize Lie subgroups in infinite dimensions, see [Neeb, 2006, Theorem IV.3.3] (even for certain classes of Lie groups modeled on locally convex spaces). Third, since $\widetilde{\exp }: \widetilde{\mathrm{L}}(\mathbb{R}) \rightarrow \widetilde{\mathrm{L}}\left(S^{1}\right)$ maps bijectively each connected component of $\widetilde{\mathrm{L}}(\mathbb{R})$ to a connected component of $\widetilde{\mathrm{L}}\left(S^{1}\right)$, it induces a Hilbert Lie group structure on $\widetilde{\mathrm{L}}\left(S^{1}\right)$.

The commutative Hilbert Lie algebra of $\widetilde{\mathrm{L}}\left(S^{1}\right)$ is clearly $H^{s}\left(S^{1}, \mathbb{R}\right):=\left\{u: S^{1} \rightarrow \mathbb{R} \mid u\right.$ of class $\left.H^{s}\right\}$, the space of periodic $H^{s}$ maps, and the exponential map exp: $H^{s}\left(S^{1}, \mathbb{R}\right) \rightarrow \widetilde{\mathrm{L}}\left(S^{1}\right)$ is given by $\exp (u)(\theta)=e^{\mathrm{i} u(\theta)}$, where $\theta \in \mathbb{R} / 2 \pi \mathbb{Z}=S^{1}$.

\subsection{The based loop group of $S^{1}$}

The inner product on the Hilbert space $L^{2}\left(S^{1}\right)$ of $L^{2}$ real valued functions on $S^{1}$ is defined by 


$$
\langle f, g\rangle:=\frac{1}{2 \pi} \int_{-\pi}^{\pi} \mathrm{d} \theta f(\theta) g(\theta), \quad f, g \in L^{2}\left(S^{1}\right) .
$$

Following Pressley [1982] and Ativah and Pressley [1983], we introduce the closed Hilbert Lie subgroup L $\left(S^{1}\right):=$ $\left\{\varphi \in \widetilde{\mathrm{L}}\left(S^{1}\right) \mid \varphi(1)=1\right\}$ of $\widetilde{\mathrm{L}}\left(S^{1}\right)$ whose closed commutative Hilbert Lie algebra is $\mathrm{L}(\mathbb{R}):=\left\{u \in H^{s}\left(S^{1}, \mathbb{R}\right) \mid u(1)=0\right\}$. The exponential map exp : $\mathrm{L}(\mathbb{R}) \ni u \mapsto e^{\mathrm{i} u} \in \mathrm{L}\left(S^{1}\right)$ is a Lie group isomorphism (with $\mathrm{L}(\mathbb{R})$ thought of as a commutative group relative to addition), a fact that will play a very important role later on (see also [Pressley and Segal, 1986, page $151, \S 8.9])$.

There is a natural 2-cocycle $\omega$ on $\mathrm{L}(\mathbb{R})$, namely

$$
\omega(u, v):=\frac{1}{2 \pi} \int_{-\pi}^{\pi} \mathrm{d} \theta u^{\prime}(\theta) v(\theta)=\left\langle u^{\prime}, v\right\rangle
$$

where $u^{\prime}:=d u / d \theta$. Therefore, there is a central extension of Lie algebras

$$
0 \longrightarrow \mathbb{R} \longrightarrow \widehat{\mathrm{L}(\mathbb{R})} \longrightarrow \mathrm{L}(\mathbb{R}) \longrightarrow 0
$$

which, as shown in Segal [1981], integrates to a central extension of Lie groups

$$
1 \longrightarrow S^{1} \longrightarrow \widehat{\mathrm{L}\left(S^{1}\right)} \longrightarrow \mathrm{L}\left(S^{1}\right) \longrightarrow 1 .
$$

The "geometric duals" of $\mathrm{L}(\mathbb{R})$ and $\widehat{\mathrm{L}(\mathbb{R})}=\mathbb{R} \oplus \mathrm{L}(\mathbb{R})$ are themselves, relative to the weak $L^{2}$-pairing. It turns out that the coadjoint action of $\widehat{\mathrm{L}\left(S^{1}\right)}$ on $\widehat{\mathrm{L}(\mathbb{R})}$ preserves $\{1\} \oplus \mathrm{L}(\mathbb{R})$ so that, as usual, the coadjoint action of $\widehat{\mathrm{L}\left(S^{1}\right)}$ on $\mathrm{L}(\mathbb{R})$ is an affine action which, in this case, because the group is commutative, equals

$$
\operatorname{Ad}_{e^{i f}}^{*} \mu=\frac{f^{\prime}}{f}=(\log |f|)^{\prime} \quad e^{\mathrm{i} f} \in \mathrm{L}\left(S^{1}\right), \quad \mu \in \mathrm{L}(\mathbb{R}) .
$$

Thus, the orbit of the constant function 0 is $\widehat{\mathrm{L}\left(S^{1}\right)} / S^{1}$ (where the denominator is thought of as constant loops), i.e., it equals $\mathrm{L}\left(S^{1}\right)$. Therefore, every element $u \in \mathrm{L}(\mathbb{R})$ of its Lie algebra has, in Fourier representation, vanishing zero order Fourier coefficient, i.e., $\widehat{u}(0)=0$.

Thus, the based loop group is a coadjoint orbit of its natural central extension and, according to 2 has three distinguished weak Riemannian metrics. These were computed explicitly in Pressley [1982], Atiyah and Pressley [1983], Pressley and Segal [1986]; we recall them below.

\section{3 $\mathrm{L}\left(S^{1}\right)$ as a weak Kähler manifold}

Note that on $L(\mathbb{R})$, the cocycle $(15)$ is weakly non-degenerate. Therefore, left (or right) translating it at every point of the group $\mathrm{L}\left(S^{1}\right)$ yields a weakly non-degenerate closed two-form, i.e., a symplectic form. Thus, as expected, since it is a coadjoint orbit, the Hilbert Lie group $\mathrm{L}\left(S^{1}\right)$ carries an invariant symplectic form whose value at the identity element 1 (the constant loop equal to 1) is given by 15 .

Now we introduce the Hilbert transform on the circle

$$
\mathscr{H} u(\theta):=\frac{1}{2 \pi} f_{-\pi}^{\pi} \mathrm{d} s u(s) \cot \left(\frac{\theta-s}{2}\right)=\frac{1}{2 \pi} \int_{-\pi}^{\pi} \mathrm{d} s u(\theta-s) \cot \left(\frac{s}{2}\right):=\lim _{\varepsilon \rightarrow 0+} \frac{1}{\pi} \int_{\varepsilon \leq|s| \leq \pi} \mathrm{d} s u(\theta-s) \cot \left(\frac{s}{2}\right)
$$

for any $u \in L^{2}\left(S^{1}\right)$, where $f$ denotes the Cauchy principal value. We adopt here the sign conventions in $[$ King, 2009, Formulas (3.202) and (6.38), Vol. 1]. If $u \in L^{2}\left(S^{1}\right)$, then $\mathscr{H} u \in L^{2}\left(S^{1}\right)$ and it is defined for almost every $\theta \in[-\pi, \pi]$ (Lusin's Theorem, [King, 2009, §6.19, Vol. 1]). The Hilbert transform has the following remarkable properties that will be used later on:

- If $u(\theta)=\sum_{n=-\infty}^{\infty} \widehat{u}(n) e^{\mathrm{i} n \theta} \in L^{2}\left(S^{1}\right)$, where $\widehat{u}(n):=\frac{1}{2 \pi} \int_{-\pi}^{\pi} \mathrm{d} \theta u(\theta) e^{-\mathrm{i} n \theta}$, so $\overline{\widehat{u}(n)}=\widehat{u}(-n)$ since $u$ is real valued, then 


$$
\mathscr{H} u(\theta)=-\mathrm{i} \sum_{n=-\infty}^{\infty} \widehat{u}(n) \operatorname{sign}(n) e^{\mathrm{i} n \theta} \in L^{2}\left(S^{1}\right)
$$

which follows from the identity $\widehat{\mathscr{H}} f(n)=-\mathrm{i} \widehat{f}(n) \operatorname{sign}(n)$ (King, 2009, Formulas (6.100) or (6.124), Vol. 1]). Here, $\operatorname{sign}(n)=1$ if $n \in \mathbb{N}, \operatorname{sign}(n)=-1$ if $n \in-\mathbb{N}$, and $\operatorname{sign}(0)=0$. Note that $\mathscr{H} u$ is also real valued since $\widehat{u}(n) \operatorname{sign}(n)=$ $-\widehat{u}(-n) \operatorname{sign}(-n)$. The formula above implies that ([King, 2009, Formula (6.126), Vol. 1])

$$
\int_{-\pi}^{\pi} \mathrm{d} s \mathscr{H} u(s)=0
$$

- For every $u \in L^{2}\left(S^{1}\right)$, we have the orthogonality property ([King, 2009, Formula (6.127), Vol. 1]):

$$
\langle u, \mathscr{H} u\rangle=0 .
$$

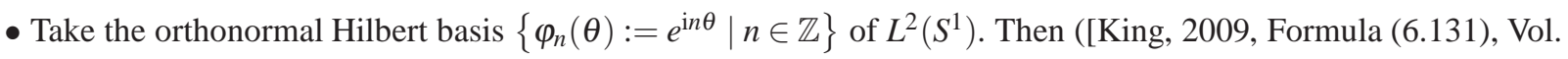
1]):

$$
\mathscr{H} \varphi_{n}(\theta)=-\mathrm{i} \operatorname{sign}(n) \varphi_{n}(\theta), \quad \text { for all } n \in \mathbb{Z} .
$$

So, the eigenvalues of $\mathscr{H}$ are: $-\mathrm{i}$ for all $n>0$, i for all $n<0$, and 0 if $n=0$.

- If $u, v \in L^{2}\left(S^{1}\right)$ then ([King, 2009, Formula (6.99), Vol. 1])

$$
\langle u, v\rangle=\frac{1}{4 \pi^{2}}\left(\int_{-\pi}^{\pi} \mathrm{d} s u(s)\right)\left(\int_{-\pi}^{\pi} \mathrm{d} s v(s)\right)+\langle\mathscr{H} u, \mathscr{H} v\rangle
$$

and hence (King, 2009, Formula (6.97), Vol. 1])

$$
\|u\|_{L^{2}\left(S^{1}\right)}^{2}=\left(\frac{1}{2 \pi} \int_{-\pi}^{\pi} \mathrm{d} s u(s)\right)^{2}+\|\mathscr{H} u\|_{L^{2}\left(S^{1}\right)}^{2}
$$

for any $u \in L^{2}\left(S^{1}\right)$. This shows that $\|\mathscr{H} u\|_{L^{2}\left(S^{1}\right)}^{2} \leq\|u\|_{L^{2}\left(S^{1}\right)}^{2}$ and the constant 1 is the best possible ([King, 2009, Formulas (6.167) and (6.168), Vol. 1]). In particular, if the average of $u$ is zero, then $\mathscr{H}$ is an isometry of $L^{2}\left(S^{1}\right)$.

- The Hilbert transform is skew-adjoint relative to the $L^{2}\left(S^{1}\right)$-inner product, i.e., $\mathscr{H}^{*}=-\mathscr{H}$ ([King, 2009, Formula (6.98) or (6.106), Vol. 1]).

- For any $u \in L^{2}\left(S^{1}\right)$ we have ([King, 2009, Formula (6.34), (6.82), or (6.156), Vol. 1]):

$$
\mathscr{H}^{2} u(\theta)=-u(\theta)+\frac{1}{2 \pi} \int_{-\pi}^{\pi} \mathrm{d} s u(s)=-u(\theta)+\widehat{u}(0) .
$$

- For any $u \in H^{s}\left(S^{1}\right)$ with $s \geq 0$ we have $\mathscr{H} u \in H^{s}\left(S^{1}\right)$; this is an immediate consequence of (17). If $s \geq 1$, then $\mathscr{H} u^{\prime}=(\mathscr{H} u)^{\prime}$, i.e., $\mathscr{H} \circ \frac{d}{d \theta}=\frac{\bar{d}}{d \theta} \circ \mathscr{H}$ on $H^{s}\left(S^{1}\right)$ with $s \geq 1$.

Using these properties, if $u(\theta)=\sum_{n=-\infty}^{\infty} \widehat{u}(n) e^{\mathrm{i} n \theta} \in H^{1}\left(S^{1}\right)$, then $u^{\prime}(\theta)=\sum_{n=-\infty}^{\infty} \widehat{u}(n)$ in $e^{\mathrm{i} n \theta} \in L^{2}\left(S^{1}\right)$ and hence

$$
\left(\mathscr{H} u^{\prime}\right)(\theta)=(\mathscr{H} u)^{\prime}(\theta)=\left(-\mathrm{i} \sum_{n=-\infty}^{\infty} \widehat{u}(n) \operatorname{sign}(n) e^{\mathrm{i} n \theta}\right)^{\prime}=\sum_{n=-\infty}^{\infty}|n| \widehat{u}(n) e^{i n \theta}
$$

On the other hand, if $v \in H^{2}\left(S^{1}\right)$, then

$$
-\frac{d^{2}}{d \theta^{2}} v(\theta)=\sum_{n=-\infty}^{\infty} n^{2} \widehat{v}(n) e^{i n \theta}
$$

and hence if $u \in H^{1}\left(S^{1}\right)$,

$$
\left(-\frac{d^{2}}{d \theta^{2}}\right)^{\frac{1}{2}} u(\theta)=\sum_{n=-\infty}^{\infty}|n| \widehat{u}(n) e^{i n \theta}=\left(\mathscr{H} u^{\prime}\right)(\theta)=\left(\left(\mathscr{H} \circ \frac{d}{d \theta}\right) u\right)(\theta)
$$


by (18). By the previous properties we have $(\mathscr{H} \circ d / d \theta)^{2}=-d^{2} / d \theta^{2}$, as expected; note that the the extra term, which is the zero order Fourier coefficient, does not appear in this case, because the derivative eliminates it.

Now, if $\varphi=e^{\mathrm{i} f} \in \mathrm{L}\left(S^{1}\right)$, i.e., $\varphi(1)=1$ and $f:[-\pi, \pi] \rightarrow \mathbb{R}$ is a periodic function, then $\widehat{f}(0)=f(0)=0$. Similarly, if $u \in \mathrm{L}(\mathbb{R})$, i.e., $u(1)=0$ and we think of $u$ as a periodic function $u:[-\pi, \pi] \rightarrow \mathbb{R}$, then $\widehat{u}(0)=u(0)=0$. This, and the properties of the Hilbert transform on the circle, imply: $\mathscr{H}(\mathrm{L}(\mathbb{R})) \subseteq \mathrm{L}(\mathbb{R}), \mathscr{H}$ is unitary on $\mathrm{L}(\mathbb{R})$ (relative to the $H^{s}$-inner product), $\mathscr{H} \circ \mathscr{H}=-I$ on $\mathrm{L}(\mathbb{R})$. Concretely, the Hilbert transform on $\mathrm{L}(\mathbb{R})$ has the form:

$$
u(\theta)=\sum_{n \in \mathbb{Z} \backslash\{0\}} \widehat{u}(n) e^{\mathrm{i} n \theta} \in \mathrm{L}(\mathbb{R}) \quad \Longrightarrow \quad \mathscr{H} u(\theta)=-\mathrm{i} \sum_{n \in \mathbb{Z} \backslash\{0\}} \widehat{u}(n) \operatorname{sign}(n) e^{\mathrm{i} n \theta} \in \mathrm{L}(\mathbb{R}) .
$$

Thus, $\mathscr{H}$ defines the structure of a complex Hilbert space on $\mathrm{L}(\mathbb{R})$, relative to the $H^{s}$ inner product, $s \geq 1$. Hence, translating $\mathscr{H}$ to any tangent space of $\mathrm{L}\left(S^{1}\right)$, we obtain an invariant almost complex structure on the Hilbert Lie group $\mathrm{L}\left(S^{1}\right)$ which is, in fact, a complex structure. For general criteria how to obtain complex structures on real Banach manifolds, see Beltita [2005]; the argument above is a very special case of these general methods.

Finally, $\mathrm{L}\left(S^{1}\right)$ is a Kähler manifold, as proved in Atiyah and Pressley [1983]. This is immediately seen by noting that

$$
g(1)(u, v):=\omega(\mathscr{H} u, v)=\sum_{n=-\infty}^{\infty}|n| \widehat{u}(n) \widehat{v}(n)
$$

is symmetric and positive definite and so, by translations, defines a weak Riemannian metric on $\mathrm{L}\left(S^{1}\right)$. Note that this metric is not the $H^{s}$ metric for any $s \geq 1$. In fact, the metric $g$ is incomplete, whereas the $H^{s}$ metric is complete.

Concluding, $\left(\mathrm{L}\left(S^{1}\right), \omega, g, \mathscr{H}\right)$ is a weak Kähler manifold and all structures are group invariant (see Pressley [1982], Atiyah and Pressley [1983], Pressley and Segal [1986]).

\subsection{Weak Riemannian metrics on $\mathrm{L}\left(S^{1}\right)$}

The three metrics discussed in $\S 2$ for $\mathrm{L}\left(S^{1}\right)$, viewed as a coadjoint orbit of its central extension, have been computed by Pressley [1982]. We recall here relevant formulas.

The induced metric is defined by the natural inner product on $L(\mathbb{R})$, which is the usual $L^{2}$-inner product. Hence, the induced metric is obtained by left (equivalently, right) translation of the inner product

$$
b(1)(u, v):=\langle u, v\rangle=\frac{1}{2 \pi} \int_{-\pi}^{\pi} \mathrm{d} t u(t) v(t)
$$

for any two functions $u, v \in \mathrm{L}(\mathbb{R})$.

Define the following inner products on $\mathrm{L}(\mathbb{R})$ :

$$
\begin{aligned}
& b_{2}(1)(u, v):=b(1)\left(u, \mathscr{H} v^{\prime}\right)=\left\langle u, \mathscr{H} v^{\prime}\right\rangle, \quad \text { if } \quad u, v \in H^{s}\left(S^{1}\right), s \geq 1 \\
& b_{1}(1)(u, v):=b(1)\left(u^{\prime}, v^{\prime}\right)=\left\langle u^{\prime}, v^{\prime}\right\rangle, \quad \text { if } \quad u, v \in H^{s}\left(S^{1}\right), s \geq 1 .
\end{aligned}
$$

Bilinearity and symmetry of $b_{1}(1)$ and $b_{2}(1)$ are obvious. If $u \in \mathrm{L}\left(S^{1}\right)$, writing $u(\theta)=\sum_{n=-\infty}^{\infty} \widehat{u}(n) e^{\mathrm{i} n \theta}$ with $\widehat{u}(0)=0$, we have $u^{\prime}(\theta)=\mathrm{i} \sum_{n=-\infty}^{\infty} n \widehat{u}(n) e^{i n \theta}$. Since $\left\{e^{i n \theta} \mid n \in \mathbb{Z}\right\}$ is an orthonormal Hilbert basis of $L^{2}\left(S^{1}\right)$, we get

$$
b_{1}(1)(u, u)=\sum_{n=-\infty}^{\infty} n^{2}|\widehat{u}(n)|^{2} \geq 0 .
$$

In addition, $b_{1}(1)(u, u)=0$ if and only if $\widehat{u}(n)=0$ for all $n \neq 0$, i.e., $u(\theta)=\widehat{u}(0)=0$. This shows that $b_{1}(1)$ is indeed an inner product on $\mathrm{L}(\mathbb{R})$ which coincides with the $H^{1}$ inner product. Hence, if $\mathrm{L}(\mathbb{R})$ is endowed with the $H^{s}$ topology for $s \geq 1$, this inner product is strong if $s=1$ and weak if $s>1$. Left translating this inner product to any tangent space of $\mathrm{L}\left(S^{1}\right)$ (endowed with the $H^{s}$ topology for $s \geq 1$ ), yields a Riemannian metric on $\mathrm{L}\left(S^{1}\right)$ that is strong for $s=1$ and weak for $s>1$. This Riemannian metric is the normal metric on $\mathrm{L}\left(S^{1}\right)$.

The inner product $b_{2}(1)$ is identical to $g(1)$ by (21), 23), and 15). Thus, translating this inner product to the tangent space at every point of the Hilbert Lie group $\mathrm{L}\left(S^{1}\right)$, yields the standard Kähler metric $b_{2}=g$ on $\mathrm{L}\left(S^{1}\right)$, endowed with 
the $H^{s}$ topology for $s \geq 1$. Note that if $u \in \mathrm{L}\left(S^{1}\right)$, then

$$
b_{2}(1)(u, u)=\sum_{n=-\infty}^{\infty}|n||\widehat{u}(n)|^{2}
$$

which shows that the Kähler metric $b_{2}$ coincides with the $H^{1 / 2}$ metric and is, therefore, a weak metric on $\mathrm{L}\left(S^{1}\right)$.

There are relations similar to (13) and (14), namely

$$
b(1)(u, v)=b_{1}(1)\left(\mathscr{A}^{2} u, v\right), \quad b_{2}(1)(u, v)=b_{1}(1)(\mathscr{A} u, v),
$$

where

$$
\left(\mathscr{A}^{2} u\right)(\theta)=\sum_{n=-\infty}^{\infty} n^{2} \widehat{u}(n) e^{\mathrm{i} n \theta}, \quad(\mathscr{A} u)(\theta)=\sum_{n=-\infty}^{\infty}|n| \widehat{u}(n) e^{\mathrm{i} n \theta}
$$

if $u(\theta)=\sum_{n=-\infty}^{\infty} \widehat{u}(n) e^{\mathrm{i} n \theta}$. However, note that the relation involving $\mathscr{A}^{2}$ requires that $u \in H^{s}\left(S^{1}\right)$ with $s \geq 2$.

\subsection{Vector fields on $\mathrm{L}\left(S^{1}\right)$ and $\mathrm{L}(\mathbb{R})$}

Recall that the exponential map exp : $\mathrm{L}(\mathbb{R}) \ni u \mapsto e^{\mathrm{i} u} \in \mathrm{L}\left(S^{1}\right)$ is a Lie group isomorphism ([Pressley and Segal, 1986, page 151, §8.9]). Here, we identified the Lie algebra of $S^{1}$ with $\mathbb{R}$, even though, naturally, it is the imaginary axis, the tangent space at $1 \in S^{1}$ to $S^{1}$. This means that care must be taken when carrying out standard Lie group operations with the exponential map, interpreted as the exponential of a purely imaginary number. Since such computations affect our next results, we clarify these statements below.

The tangent space at the identity 1 to $S^{1}$ is the imaginary axis. This is the natural Lie algebra of the Lie group $S^{1}$ and the exponential map is given by exp : $\mathrm{i} \mathbb{R} \ni(\mathrm{i} x) \mapsto e^{\mathrm{i} x} \in S^{1}$. Of course, traditionally, one identifies $\mathrm{i} \mathbb{R}$ with $\mathbb{R}$ by dividing by $i$ and thinks of the exponential map as exp : $\mathbb{R} \ni x \mapsto e^{\mathrm{i} x} \in S^{1}$. Unfortunately, this induces some problems. For example, since (left) translation is given by $L_{e^{i x}} e^{\mathrm{iy}}:=e^{\mathrm{i} x} e^{\mathrm{i} y}$, it follows that

$$
T_{1} L_{e^{\mathrm{i} x}}(\mathrm{i} y):=\left.\frac{d}{d \varepsilon}\right|_{\varepsilon=0} L_{e^{\mathrm{i} x}} e^{\mathrm{i} \varepsilon y}=\left.\frac{d}{d \varepsilon}\right|_{\varepsilon=0} e^{\mathrm{i} x} e^{\mathrm{i} \varepsilon y}=\mathrm{i} y e^{\mathrm{i} x},
$$

so the identification of the Lie algebra with $\mathbb{R}$ poses no problems and we have, dividing both sides by $\mathrm{i}$,

$$
T_{1} L_{e^{\mathrm{ix}}}(y)=y e^{\mathrm{i} x} .
$$

However, the definition of the exponential map for any Lie group $G$ with Lie algebra $\mathfrak{g}$, yields

$$
\frac{d}{d t} \exp (t \xi)=T_{e} L_{\exp (t \xi)} \xi, \quad \text { for all } \xi \in \mathfrak{g} .
$$

This formula works perfectly well if the Lie algebra of $S^{1}$ is i $\mathbb{R}$. Indeed

$$
\frac{d}{d t} e^{t i x}=\mathrm{i} x e^{t i x}
$$

which coincides with (27) in view of (25). On the other hand, if the Lie algebra is thought of as $\mathbb{R}$, i.e., the right hand side needs to be divided by $\mathrm{i}$, then with the definition of $\exp (t x)=e^{\mathrm{i} t x}$ the identity above is no longer valid. What we should get is

$$
\frac{d}{d t} \exp (t x)=x \exp (t x)=T_{1} L_{\exp (t x)} x=x e^{\mathrm{i} t x}
$$

by (26) if $\exp (t x)=e^{\mathrm{i} t x}$, but the right hand side gives ix $e^{\mathrm{i} t x}$, as we saw above. In other words, if the Lie algebra of $S^{1}$ is thought of as $\mathbb{R}$, as is traditionally done, then we need a formula for the derivative of the Lie group exponential map in terms of the exponential map of purely imaginary numbers. In view of the previous discussion, this formula is 


$$
\frac{d}{d t} \exp (t x):=\frac{1}{\mathrm{i}} \frac{d}{d t} e^{\mathrm{i} t x}=x e^{\mathrm{i} t x}
$$

With these remarks in mind, we shall now compute the push-forward of a vector field on $\mathrm{L}(\mathbb{R})$ to $\mathrm{L}\left(S^{1}\right)$.

Proposition 2. Let $X \in \mathfrak{X}(\mathrm{L}(\mathbb{R}))$ be an arbitrary vector field. Then its push-forward to $\left.\mathrm{L}\left(S^{1}\right)\right)$ has the expression

$$
\left(\exp _{*} X\right)\left(e^{\mathrm{i} u}\right)=X(u) e^{\mathrm{i} u}
$$

for any $u \in \mathrm{L}(\mathbb{R})$.

Proof. By the definition of push forward of vector fields by a diffeomorphism, we have

$$
\begin{aligned}
\left(\exp _{*} X\right)\left(e^{\mathrm{i} u}\right) & =\left(T \exp \times \circ \circ \exp ^{-1}\right)\left(e^{\mathrm{i} u}\right)=T_{u} \exp (X(u))=\left.\frac{d}{d \varepsilon}\right|_{\varepsilon=0} \exp (u+\varepsilon X(u)) \\
& =\left.\frac{d}{d \varepsilon}\right|_{\varepsilon=0} \exp (u) \exp (\varepsilon X(u))=\left(\left.\frac{d}{d \varepsilon}\right|_{\varepsilon=0} \exp (\varepsilon X(u))\right) \exp (u) \stackrel{28}{=}\left(\left.\frac{1}{\mathrm{i}} \frac{d}{d \varepsilon}\right|_{\varepsilon=0} e^{i \varepsilon X(u)}\right) e^{\mathrm{i} u} \\
& =X(u) e^{\mathrm{i} u}
\end{aligned}
$$

as stated.

\subsection{The gradient vector fields in the three metrics of $\mathrm{L}\left(S^{1}\right)$}

We compute now the gradients of a specific function using the three metrics.

Theorem 1. The gradients of the smooth function $H: \mathrm{L}\left(S^{1}\right) \rightarrow \mathbb{R}$ given by

$$
H\left(e^{\mathrm{i} f}\right)=\frac{1}{4 \pi} \int_{-\pi}^{\pi} \mathrm{d} \theta f^{\prime}(\theta)^{2}
$$

are

(i) $\quad \nabla^{1} H\left(e^{\mathrm{i} f}\right)=f e^{\mathrm{i} f}$ for the normal metric $b_{1}$;

(ii) $\nabla H\left(e^{\mathrm{i} f}\right)=-f^{\prime \prime} e^{\mathrm{i} f}$ with respect to the induced metric $b$ for $f \in H^{s}\left(S^{1}\right)$ with $s \geq 2$;

(iii) $\nabla^{2} H\left(e^{\mathrm{i} f}\right)=\left(\mathscr{H} f^{\prime}\right) e^{\mathrm{i} f}$ with respect to the weak Kähler metric $b_{2}$.

Proof. (i) Since $T_{1} L_{e^{\mathrm{i} f}} u=u e^{\mathrm{i} f}$ for any $u \in \mathrm{L}(\mathbb{R})$ and $e^{\mathrm{i} f} \in \mathrm{L}\left(S^{1}\right)$, invariance of $b_{1}$ yields

$$
\begin{aligned}
b_{1}(1)\left(e^{-\mathrm{i} f} \nabla^{1} H\left(e^{\mathrm{i} f}\right), u\right) & =b_{1}\left(e^{\mathrm{i} f}\right)\left(\nabla^{1} H\left(e^{\mathrm{i} f}\right), u e^{\mathrm{i} f}\right)=\mathbf{d} H\left(e^{\mathrm{i} f}\right)\left(u e^{\mathrm{i} f}\right) \\
& =\left.\frac{d}{d t}\right|_{t=0} H\left(e^{\mathrm{i}(f+t u)}\right)=\left.\frac{d}{d t}\right|_{t=0} \frac{1}{4 \pi} \int_{-\pi}^{\pi} \mathrm{d} \theta\left(f^{\prime}(\theta)+t u^{\prime}(\theta)\right)^{2} \\
& =\frac{1}{2 \pi} \int_{-\pi}^{\pi} \mathrm{d} \theta f^{\prime}(\theta) u^{\prime}(\theta)=\left\langle f^{\prime}, u^{\prime}\right\rangle b_{1}(1)(f, u)
\end{aligned}
$$

which shows that $\nabla^{1} H\left(e^{\mathrm{i} f}\right)=f e^{\mathrm{i} f}$.

(ii) Proceeding as above, using the same notations, and assuming that $f \in H^{s}\left(S^{1}\right)$ with $s \geq 2$, we have

$$
\begin{aligned}
b(1)\left(e^{-\mathrm{i} f} \nabla H\left(e^{\mathrm{i} f}\right), u\right) & =b\left(e^{\mathrm{i} f}\right)\left(\nabla H\left(e^{\mathrm{i} f}\right), u e^{\mathrm{i} f}\right)=\mathbf{d} H\left(e^{\mathrm{i} f}\right)\left(u e^{\mathrm{i} f}\right) \\
& =\frac{1}{2 \pi} \int_{-\pi}^{\pi} \mathrm{d} \theta f^{\prime}(\theta) u^{\prime}(\theta)=-\frac{1}{2 \pi} \int_{-\pi}^{\pi} \mathrm{d} \theta f^{\prime \prime}(\theta) u(\theta) \\
& =\left\langle-f^{\prime \prime}, u\right\rangle \stackrel{12 .}{=} b(1)\left(-f^{\prime \prime}, u\right)
\end{aligned}
$$

which shows that $\nabla H\left(e^{\mathrm{i} f}\right)=-f^{\prime \prime} e^{\mathrm{i} f}$. 
(iii) This computation uses the isometry property of $\mathscr{H}$ relative to the $L^{2}$ inner product. We have,

$$
\begin{aligned}
b_{2}(1)\left(e^{-\mathrm{i} f} \nabla^{2} H\left(e^{\mathrm{i} f}\right), u\right) & =b_{2}\left(e^{\mathrm{i} f}\right)\left(\nabla^{2} H\left(e^{\mathrm{i} f}\right), u e^{\mathrm{i} f}\right)=\mathbf{d} H\left(e^{\mathrm{i} f}\right)\left(u e^{\mathrm{i} f}\right) \\
& =\left\langle f^{\prime}, u^{\prime}\right\rangle=\left\langle\mathscr{H} f^{\prime}, \mathscr{H} u^{\prime}\right\rangle \stackrel{23}{=} b_{2}(1)\left(\mathscr{H} f^{\prime}, u\right)
\end{aligned}
$$

which shows that $\nabla^{2} H\left(e^{\mathrm{i} f}\right)=\left(\mathscr{H} f^{\prime}\right) e^{\mathrm{i} f}$.

Since

$$
\omega\left(e^{\mathrm{i} f}\right)\left(\mathscr{H} \nabla^{2} H\left(e^{\mathrm{i} f}\right), u e^{\mathrm{i} f}\right) \stackrel{21}{=} b_{2}\left(e^{\mathrm{i} f}\right)\left(\nabla^{2} H\left(e^{\mathrm{i} f}\right), u e^{\mathrm{i} f}\right)=\mathbf{d} H\left(e^{\mathrm{i} f}\right)\left(u e^{\mathrm{i} f}\right)
$$

it follows that the Hamiltonian vector field on $\left(\mathrm{L}\left(S^{1}\right), \omega\right)$ for the function $H$ is $X_{H}=\mathscr{H} \nabla^{2} H$. Since $\mathscr{H}$ commutes with the tangent lift to group translations, Theorem 1 (iii) implies that

$$
X_{H}\left(e^{\mathrm{i} f}\right)=\left(\mathscr{H} \nabla^{2} H\right)\left(e^{\mathrm{i} f}\right)=\mathscr{H}\left(\nabla^{2} H\left(e^{\mathrm{i} f}\right)\right)=\mathscr{H}\left(\left(\mathscr{H} f^{\prime}\right) e^{\mathrm{i} f}\right)=-f^{\prime} e^{\mathrm{i} f}
$$

This proves the first part of the following statement.

Corollary 1. The Hamiltonian vector field of $H$ relative to the translation invariant symplectic form $\omega$ on $\mathrm{L}\left(S^{1}\right)$ whose value at the identity element is given by (15) has the expression $X_{H}\left(e^{\mathrm{i} f}\right)=-f^{\prime} e^{\mathrm{i} f}$. Its flow is the rotation

$$
\left(F_{t}\left(e^{\mathrm{i} f}\right)\right)(\theta)=e^{-\mathrm{i}(f(t+\theta)-f(t))}
$$

Proof. Since $\mathrm{L}(\mathbb{R}) \ni u \longmapsto e^{\mathrm{i} u} \in \mathrm{L}\left(S^{1}\right)$ is the exponential map and we think of $\mathbb{R}$ as the Lie algebra of $S^{1}$ (and not the imaginary axis), we write $d e^{\mathrm{i} t u} / d t=u e^{\mathrm{i} t u}$ without the factor of $\mathrm{i}$ in front (see (28)). The verification that $F_{t}$ is indeed the flow of $X_{H}$ is straightforward:

$$
\begin{aligned}
\frac{d}{d t}\left(F_{t}\left(e^{\mathrm{i} f}\right)\right)(\theta) & =\frac{d}{d t} e^{-\mathrm{i}(f(t+\theta)-f(t))}=-\left(f^{\prime}(t+\theta)-f^{\prime}(t)\right) e^{-\mathrm{i}(f(t+\theta)-f(t))} \\
& =X_{H}\left(F_{t}\left(e^{\mathrm{i} f}\right)\right)(\theta)
\end{aligned}
$$

as required.

We recover thus [Pressley, 1982, Proposition 3.1] (up to a sign which is due to different conventions calibrating $\omega$, $\mathscr{H}$, and $\left.b_{2}\right)$.

Applying Proposition 2 to Theorem 1 we get the following result:

Corollary 2. The three gradient vector fields for the smooth function $H_{1}: \mathrm{L}(\mathbb{R}) \rightarrow \mathbb{R}$ given by

$$
H_{1}(u)=\frac{1}{4 \pi} \int_{-\pi}^{\pi} \mathrm{d} \theta\left(u^{\prime}\right)^{2}
$$

are

(i) $\nabla^{1} H_{1}(u)=u$ for the weak inner product $b_{1}(1)$ defining the normal metric;

(ii) $\nabla H_{1}(u)=-u^{\prime \prime}$ for the weak inner product $b(1)$ defining the induced metric, where for $u \in H^{s}(\mathbb{R})$ with $s \geq 2$;

(iii) $\nabla^{2} H_{1}(u)=\mathscr{H} u^{\prime}$ for the weak inner product $b_{2}(1)$ defining the Kähler metric.

Since the exponential map is a Lie group isomorphism and the three metrics coincide with the respective inner products at the identity, their left invariance guarantees that the three inner products on $\mathrm{L}(\mathbb{R})$ correspond to the three invariant metrics on $\mathrm{L}\left(S^{1}\right)$.

Applying Proposition 2 to Corollary 1, we conclude:

Corollary 3. The Hamiltonian vector field of $H_{1}$ relative to the symplectic form $\omega$ given by (15) has the expression $X_{H}(u)=-u^{\prime}$. Its flow is $\left(F_{t}(u)\right)(\theta)=u(\theta-t)$. 
The verification of the statement about the flow is immediate:

$$
\frac{d}{d t}\left(F_{t}(u)\right)(\theta)=\frac{d}{d t} u(\theta-t)=-u^{\prime}(\theta-t)=\left(X_{H}\left(F_{t}(u)\right)\right)(\theta)
$$

If one is willing to put more stringent hypotheses on the functional, it is possible to obtain a general result.

Theorem 2. Let $H: \mathrm{L}\left(S^{1}\right) \rightarrow \mathbb{R}$ be a smooth function (with $\mathrm{L}\left(S^{1}\right)$ endowed, as usual, with the $H^{s}$ topology for $s \geq 1$ ) and assume that the functional derivative $\delta H / \delta u \in \mathrm{L}\left(S^{1}\right)$ exists. Then the gradient vector fields are

(i) $\nabla H(u)=\frac{\delta H}{\delta u}$ with respect the weak inner product $b(1)$ defining the induced metric;

(ii) $\left(\nabla^{1} H(u)\right)(\theta)=-\int_{0}^{\theta} \mathrm{d} \varphi\left(\int_{0}^{\varphi} \mathrm{d} \psi \frac{\delta H}{\delta u}(\psi)\right)$ with respect to the (weak) inner product $b_{1}(1)$ defining the normal metric, provided both $\int_{0}^{\theta} \mathrm{d} \varphi \frac{\delta H}{\delta u}(\varphi)$ and $\int_{0}^{\theta} \mathrm{d} \varphi\left(\int_{0}^{\varphi} \mathrm{d} \psi \frac{\delta H}{\delta u}(\psi)\right)$ are periodic;

(iii) $\left(\nabla^{2} H(u)\right)(\theta)=-\mathscr{H} \int_{0}^{\theta} \mathrm{d} \varphi \frac{\delta H}{\delta u}(\varphi)$ with respect to the weak inner product $b_{2}(1)$ defining the Kähler metric, provided $\int_{0}^{\theta} \mathrm{d} \varphi \frac{\delta H}{\delta u}(\varphi)$ is periodic.

Proof. (i) For the inner product $b(1)$ on $\mathrm{L}\left(S^{1}\right)$ defining the induced metric, if $u, v \in \mathrm{L}(\mathbb{R})$, we have by periodicity of $u, v$,

$$
b(1)(\nabla H(u), v)=\mathbf{D} H(u) \cdot v=\left\langle\frac{\delta H}{\delta u}, v\right\rangle \stackrel{12}{=} b(1)\left(\frac{\delta H}{\delta u}, v\right) .
$$

This shows that $\nabla H(u)=\frac{\delta H}{\delta u}$.

(ii) For the inner product $b_{1}(1)$ on $\mathrm{L}\left(S^{1}\right)$ defining the normal metric, if $u, v \in \mathrm{L}(\mathbb{R})$, we have by periodicity of $\int_{0}^{\theta} \mathrm{d} \varphi \frac{\delta H}{\delta u}(\varphi)$ and $\int_{0}^{\theta} \mathrm{d} \varphi\left(\int_{0}^{\varphi} \mathrm{d} \psi \frac{\delta H}{\delta u}(\psi)\right)$,

$$
\begin{aligned}
b_{1}(1)\left(\nabla^{1} H(u), v\right) & =\mathbf{D} H(u) \cdot v=\left\langle\frac{\delta H}{\delta u}, v\right\rangle=\frac{1}{2 \pi} \int_{-\pi}^{\pi} \mathrm{d} \theta \frac{\delta H}{\delta u}(\theta) v(\theta) \\
& =\left.\frac{1}{2 \pi}\left(\int_{0}^{\theta} \mathrm{d} \varphi \frac{\delta H}{\delta u}(\varphi)\right) v(\theta)\right|_{-\pi} ^{\pi}-\frac{1}{2 \pi} \int_{-\pi}^{\pi} \mathrm{d} \theta\left(\int_{0}^{\theta} \mathrm{d} \varphi \frac{\delta H}{\delta u}(\varphi)\right) v^{\prime}(\theta) \\
& =-\frac{1}{2 \pi} \int_{-\pi}^{\pi} \mathrm{d} \theta \frac{d}{d \theta}\left(\int_{0}^{\theta} \mathrm{d} \varphi\left(\int_{0}^{\varphi} \mathrm{d} \psi \frac{\delta H}{\delta u}(\psi)\right)\right) v^{\prime}(\theta) \\
& =-\left\langle\frac{d}{d \theta}\left(\int_{0}^{\theta} \mathrm{d} \varphi\left(\int_{0}^{\varphi} \mathrm{d} \psi \frac{\delta H}{\delta u}(\psi)\right)\right), v^{\prime}\right\rangle b_{1}\left(-\int_{0}^{\theta} \mathrm{d} \varphi\left(\int_{0}^{\varphi} \mathrm{d} \psi \frac{\delta H}{\delta u}(\psi)\right), v\right)
\end{aligned}
$$

which shows that $\left(\nabla^{1} H(u)\right)(\theta)=-\int_{0}^{\theta} \mathrm{d} \varphi\left(\int_{0}^{\varphi} \mathrm{d} \psi \frac{\delta H}{\delta u}(\psi)\right)$.

(iii) For the inner product $b_{2}(1)$ on $\mathrm{L}\left(S^{1}\right)$ defining the Kähler metric, if $u, v \in \mathrm{L}(\mathbb{R})$, we have by periodicity of $\int_{0}^{\theta} \mathrm{d} \varphi \frac{\delta H}{\delta u}(\varphi)$ and the isometry property of $\mathscr{H}$,

$$
\begin{aligned}
b_{2}(1)\left(\nabla^{2} H(u), v\right) & =\mathbf{D} H(u) \cdot v=\left\langle\frac{\delta H}{\delta u}, v\right\rangle=\frac{1}{2 \pi} \int_{-\pi}^{\pi} \mathrm{d} \theta \frac{\delta H}{\delta u}(\theta) v(\theta) \\
& =\left.\frac{1}{2 \pi}\left(\int_{0}^{\theta} \mathrm{d} \varphi \frac{\delta H}{\delta u}(\varphi)\right) v(\theta)\right|_{-\pi} ^{\pi}-\frac{1}{2 \pi} \int_{-\pi}^{\pi} \mathrm{d} \theta\left(\int_{0}^{\theta} \mathrm{d} \varphi \frac{\delta H}{\delta u}(\varphi)\right) v^{\prime}(\theta) \\
& =-\left\langle\int_{0}^{\theta} \mathrm{d} \varphi \frac{\delta H}{\delta u}(\varphi), v^{\prime}\right\rangle=-\left\langle\mathscr{H} \int_{0}^{\theta} \mathrm{d} \varphi \frac{\delta H}{\delta u}(\varphi), \mathscr{H} v^{\prime}\right\rangle \stackrel{23}{=} b_{2}(1)\left(-\mathscr{H} \int_{0}^{\theta} \mathrm{d} \varphi \frac{\delta H}{\delta u}(\varphi), v\right)
\end{aligned}
$$

which shows that $\left(\nabla^{2} H(u)\right)(\theta)=-\mathscr{H} \int_{0}^{\theta} \mathrm{d} \varphi \frac{\delta H}{\delta u}(\varphi)$.

Corollary 4. Under the same hypothesis as in Theorem 2(iii), the Hamiltonian vector field of the smooth function $H: \mathrm{L}\left(S^{1}\right) \rightarrow \mathbb{R}$ relative to the symplectic form $\omega$ on $\mathrm{L}(\mathbb{R})$ given by 15 has the expression $X_{H}(u)=\int_{0}^{\theta} \mathrm{d} \varphi \frac{\delta H}{\delta u}(\varphi)$

Proof. We have $X_{H}(u)=\mathscr{H} \nabla^{2} H(u) \stackrel{(\text { iii) }}{=} \int_{0}^{\theta} \mathrm{d} \varphi \frac{\delta H}{\delta u}(\varphi)$. 
Of course, using Proposition 2 , there are immediate counterparts of Theorem 2 and Corollary 4 on the loop group $\mathrm{L}\left(S^{1}\right)$, which we shall not spell out explicitly.

The hypotheses guaranteeing the existence of the functional derivative of $H$ relative to the weakly non-degenerate $L^{2}$ pairing are quite severe. For example, the theorem can be applied to the functional $H_{1}$ in Corollary 2, but one needs additional smoothness. Indeed, the first thing to check is if this functional has a functional derivative. In fact, it does not, unless we assume that $u \in H^{s}\left(S^{1}\right)$ for $s \geq 2$, in which case we have

$$
\mathbf{D} H_{1}(u) \cdot v=\frac{1}{2 \pi} \int_{-\pi}^{\pi} \mathrm{d} s u^{\prime}(s) v^{\prime}(s)=\left.\frac{1}{2 \pi} u^{\prime}(s) v(s)\right|_{-\pi} ^{\pi}-\frac{1}{2 \pi} \int_{-\pi}^{\pi} \mathrm{d} s u^{\prime \prime}(s) v(s)=\left\langle-u^{\prime \prime}, v\right\rangle,
$$

i.e., $\delta H / \delta u=-u^{\prime \prime}$. With this additional hypothesis, the gradient flow with respect to the weak inner product $b(1)$ defining the induced metric is given by $u_{t}=-u^{\prime \prime}$.

Therefore, to continue computing the other two gradients of $H_{1}$, we need to assume that $u \in H^{s}\left(S^{1}\right)$ for $s \geq 2$. Provided this holds, to find the gradient relative to the (weak) inner product $b_{1}(1)$ defining the normal metric, we have to check that both

$$
\begin{gathered}
\int_{0}^{\theta} \mathrm{d} \varphi \frac{\delta H}{\delta u}(\varphi)=-\int_{0}^{\theta} \mathrm{d} \varphi u^{\prime \prime}(\varphi)=-u^{\prime}(\theta)+u^{\prime}(0) \\
\int_{0}^{\theta} \mathrm{d} \varphi\left(\int_{0}^{\varphi} \mathrm{d} \psi \frac{\delta H}{\delta u}(\psi)\right)=-\int_{0}^{\theta} \mathrm{d} \varphi\left(u^{\prime}(\varphi)-u^{\prime}(0)\right)=-u(\theta)+u^{\prime}(0) \theta
\end{gathered}
$$

are periodic. While the first one is periodic, the second one is not unless we assume that $u^{\prime}(0)=0$. With this additional hypothesis, the gradient is given by $u_{t}=u$. However, we know from Corollary 2 that neither $s \geq 2$, nor $u^{\prime}(0)=0$ is needed. In addition, this can also be seen directly, as follows. For any $u, v \in \mathrm{L}(\mathbb{R})$, we have

$$
b_{1}(1)\left(\nabla^{1} H(u), v\right)=\mathbf{D} H(u) \cdot v=\frac{1}{2 \pi} \int_{-\pi}^{\pi} \mathrm{d} s u^{\prime}(s) v^{\prime}(s)=\left\langle u^{\prime}, v^{\prime}\right\rangle \stackrel{\underline{\underline{M}}}{=} b_{1}(u, v)
$$

which shows that $\nabla^{1} H(u)=u$.

The same situation occurs in the computation of the third gradient. In the hypotheses of the theorem, we have

$$
\left(\nabla^{2} H(u)\right)(\theta)=-\mathscr{H} \int_{0}^{\theta} \mathrm{d} \varphi \frac{\delta H}{\delta u}(\varphi)=\mathscr{H}\left(u^{\prime}-u^{\prime}(0)\right)=\mathscr{H} u^{\prime}
$$

because the Hilbert transform of a constant is zero. Thus, the gradient flow is given in this case by

$$
u_{t}=\mathscr{H} u^{\prime} \stackrel{\underline{20}}{=}\left(-\frac{d^{2}}{d \theta^{2}}\right)^{\frac{1}{2}} u .
$$

As before, the same result can be obtained easier and without any additional hypotheses in the following way:

$$
b_{2}(1)\left(\nabla^{2} H(u), v\right)=\mathbf{D} H(u) \cdot v=\left\langle u^{\prime}, v^{\prime}\right\rangle=\left\langle\mathscr{H} u^{\prime}, \mathscr{H} v^{\prime}\right\rangle \stackrel{\sqrt[23]{=}}{=} b_{2}(1)\left(\mathscr{H} u^{\prime}, v\right) .
$$

\subsection{Symplectic structure on periodic functions}

The form of the periodic Korteweg-de Vries (KdV) equation we shall use is

$$
u_{t}-6 u u_{\theta}+u_{\theta \theta \theta}=0,
$$

where $u(t, \theta)$ is a real valued function of $t \in \mathbb{R}$ and $\theta \in[-\pi, \pi]$, periodic in $\theta$, and $u_{\theta}:=\partial u / \partial \theta$. The $\mathrm{KdV}$ equation is, of course, a famous integrable infinite dimensional Hamiltonian system. It is Hamiltonian on the Poisson manifold of all periodic functions relative to the Gardner [1971] bracket 


$$
\{F, G\}=\frac{1}{2 \pi} \int_{-\pi}^{\pi} \mathrm{d} \theta \frac{\delta F}{\delta u} \frac{d}{d \theta} \frac{\delta G}{\delta u}
$$

where

$$
F(u)=\int_{S^{1}} \mathrm{~d} \theta f\left(u, u_{\theta}, u_{\theta \theta}, \ldots\right)
$$

and similarly for $G$; the functional derivative $\delta F / \delta u$ is the usual one relative to the $L^{2}\left(S^{1}\right)$ inner product, i.e.,

$$
\frac{\delta F}{\delta u}=\frac{\partial f}{\partial u}-\frac{d}{d \theta}\left(\frac{\partial f}{\partial u_{\theta}}\right)+\frac{d^{2}}{d \theta^{2}}\left(\frac{\partial f}{\partial u_{\theta \theta}}\right)-\cdots
$$

The Hamiltonian vector field of $H(u)=\frac{1}{2 \pi} \int_{-\pi}^{\pi} \mathrm{d} \theta h\left(u, u_{\theta}, u_{\theta \theta}, \ldots\right)$ has the expression

$$
X_{H}(u)=\frac{d}{d \theta}\left(\frac{\delta H}{\delta u}\right) .
$$

For the $\mathrm{KdV}$ equation one takes

$$
H(u)=\frac{1}{2 \pi} \int_{-\pi}^{\pi} \mathrm{d} \theta\left(u^{3}+\frac{1}{2} u_{\theta}^{2}\right) .
$$

The Casimir functions of the Gardner bracket are all smooth functionals $C$ for which $\delta C / \delta u=c$ is a constant function, i.e.,

$$
C(u)=\langle c, u\rangle=\frac{1}{2 \pi} \int_{-\pi}^{\pi} \mathrm{d} \theta c u(\theta)=c \widehat{u}(0)
$$

Thus $C^{-1}(0)$ is a candidate weak symplectic leaf in the phase space of all periodic functions. The situation in infinite dimensions is not as clear as in finite dimensions, where this would be a conclusion, because there is no general stratification theorem and one cannot expect, in general, more than a weak symplectic form. However, in our case, this actually holds, as shown in Zaharov and Faddeev [1971]. Indeed,

$$
\begin{aligned}
\sigma\left(u_{1}, u_{2}\right): & =\frac{1}{4 \pi} \int_{-\pi}^{\pi} \mathrm{d} \theta\left(\int_{0}^{\theta} \mathrm{d} \varphi\left(u_{1}(\varphi) u_{2}(\theta)-u_{2}(\varphi) u_{1}(\theta)\right)\right)=\frac{1}{2 \pi} \int_{-\pi}^{\pi} \mathrm{d} \theta\left(\int_{0}^{\theta} \mathrm{d} \varphi u_{1}(\varphi)\right) u_{2}(\theta) \\
& =\left\langle\int_{0}^{\theta} \mathrm{d} \varphi u_{1}(\varphi), u_{2}\right\rangle
\end{aligned}
$$

defines a weak symplectic form on $L(\mathbb{R})$ whose formal Poisson bracket is 30 . This immediately shows that there is a tight relationship with the symplectic form $\omega$ of the complex Hilbert space $L(\mathbb{R})$, the Lie algebra of the based loop groups, given by [15, namely

$$
\sigma\left(\frac{d^{2}}{d \theta^{2}} u, v\right)=\omega(u, v)
$$

for all $u, v \in \mathrm{L}(\mathbb{R})$ of class $H^{s}, s \geq 2$. Defining

$$
\left(\frac{d}{d \theta}\right)^{-1} u:=\int_{0}^{\theta} \mathrm{d} \varphi u(\varphi)
$$

the KdV symplectic form $\sigma$ has the suggestive expression (see (28)

$$
\sigma\left(u_{1}, u_{2}\right)=\left\langle\left(\frac{d}{d \theta}\right)^{-1} u_{1}, u_{2}\right\rangle
$$

which is well defined on $H^{-\frac{1}{2}}\left(S^{1}, \mathbb{R}\right)$.

On the other hand, the Poisson bracket given by the Kähler symplectic form $[15)$ on $L(\mathbb{R})$ is

$$
\{F, G\}=\frac{1}{2 \pi} \int_{-\pi}^{\pi} \mathrm{d} \theta \frac{\delta F}{\delta u}\left(\frac{d}{d \theta}\right)^{-1} \frac{\delta G}{\delta u},
$$


which is similarly well defined on $H^{-\frac{1}{2}}$, and the Hamiltonian vector field defined by this bracket is given by Corollary 4 i.e.,

$$
u_{t}=X_{H}(u)=\left(\frac{d}{d \theta}\right)^{-1} \frac{\delta H}{\delta u} .
$$

Now, the gradient vector field for the corresponding Kähler metric, as computed in Theorem2(iii), is written as

$$
u_{t}=-\mathscr{H}\left(\frac{d}{d \theta}\right)^{-1} \frac{\delta H}{\delta u} .
$$

\section{Metriplectic Systems}

In this section we define metriplectic systems and show how to construct general classes of such systems in terms of triple brackets for both finite- and infinite-dimensional theories. We use some of the machinery developed above to address specific examples.

\subsection{Definition and consequences}

A metriplectic system consists of a smooth manifold $P$, two smooth vector bundle maps $\pi, \kappa: T^{*} P \rightarrow T P$ covering the identity, and two functions $H, S \in C^{\infty}(P)$, the Hamiltonian or total energy and the entropy of the system, such that

(i) $\quad\{F, G\}:=\langle\mathbf{d} F, \pi(\mathbf{d} G)\rangle$ is a Poisson bracket; in particular $\pi^{*}=-\pi$;

(ii) $(F, G):=\langle\mathbf{d} F, \kappa(\mathbf{d} G)\rangle$ is a positive semidefinite symmetric bracket, i.e., (,) is $\mathbb{R}$-bilinear and symmetric, so $\kappa^{*}=\kappa$, and $(F, F) \geq 0$ for every $F \in C^{\infty}(P)$;

(iii) $\{S, F\}=0$ and $(H, F)=0$ for all $F \in C^{\infty}(P) \Longleftrightarrow \pi(\mathbf{d} S)=\kappa(\mathbf{d} H)=0$.

The metriplectic dynamics of the system is given in terms of the two brackets by

$$
\frac{d}{d t} F=\{F, H+S\}+(F, H+S)=\{F, H\}+(F, S), \text { for all } F \in C^{\infty}(P),
$$

or, equivalently, as an ordinary differential equation, by

$$
\frac{d}{d t} c(t)=\pi(c(t)) \mathbf{d} H(c(t))+\kappa(c(t)) \mathbf{d} S(c(t))
$$

The Hamiltonian vector field $X_{H}:=\pi(\mathbf{d} H) \in \mathfrak{X}(P)$ represents the conservative or Hamiltonian part, whereas $Y_{S}:=$ $\kappa(\mathbf{d} S) \in \mathfrak{X}(P)$ the dissipative part of the full metriplectic dynamics (36) or 37).

As far as we know, first attempts to introduce such a structure were given in adjacent papers by Kaufman [1984] and Morrison [1984a]. (See also Kaufman and Morrison [1982].) Kaufman [1984] imposed, instead of (iii), the weaker condition $\{H, S\}=(H, S)=0$, which is enough, as will become apparent below, to deduce the First and Second Laws of Thermodynamics. In the plasma examples presented, he used (iii) for a large class of functions. All three axioms, including the degeneracy condition of (iii), were stated explicitly in Morrison [1984a] and Morrison [1984b]. The former treated the same kinetic example as Kaufman [1984] along with additional formalism, while the latter presented the metriplectic formalism for the compressible Navier-Stokes equations with entropy production. All three axioms were restated in Morrison [1986], where the terminology metriplectic was introduced and a detailed physical motivation for the introduction of (iii) is presented along with other examples such as a dissipative free rigid body equation and the Vlasov-Poisson equation with a collision term that generalizes the Landau and Balescu-Lenard equations. In Grmela and Öttinger [1997], under the name GENERIC (General Equations for Non-Equilibrium Reversible Irreversible Coupling), the same geometric structure was used to analyze many other equations; due to this paper and subsequent work of these authors, the metriplectic formalism has been popularized. For a very interesting modern application of this structure see Mielke [2011] and for further discussion about avenues for generalization see Morrison [2009]. 
The definition of metriplectic systems has three immediate important consequences. Let $c(t)$ be an integral curve of the system (37).

(1) Energy conservation:

$$
\frac{d}{d t} H(c(t))=\{H, H\}(c(t))+(H, S)(c(t))=0 .
$$

(2) Entropy production:

$$
\frac{d}{d t} S(c(t))=\{S, H\}(c(t))+(S, S)(c(t)) \geq 0 .
$$

(3) Maximum entropy principle yields equilibria: Suppose that there are $n$ functions $C_{1}, \ldots, C_{n} \in C^{\infty}(P)$ such that $\left\{F, C_{i}\right\}=\left(F, C_{i}\right)=0$ for all $F \in C^{\infty}(P)$, i.e., these functions are simultaneously conserved by the conservative and dissipative part of the metriplectic dynamics. Let $p_{0} \in P$ be a maximum of the entropy $S$ subject to the constraints $H^{-1}(h) \cap C_{1}^{-1}\left(c_{1}\right) \cap \ldots C_{n}^{-1}\left(c_{n}\right)$, for given regular values $h, c_{1}, \ldots, c_{n} \in \mathbb{R}$ of $H, C_{1}, \ldots, C_{n}$, respectively. By the Lagrange Multiplier Theorem, there exist $\alpha, \beta_{1}, \ldots, \beta_{n} \in \mathbb{R}$ such that

$$
\mathbf{d} S\left(p_{0}\right)=\alpha \mathbf{d} H\left(p_{0}\right)+\beta_{1} \mathbf{d} C_{1}\left(p_{0}\right)+\cdots+\mathbf{d} C_{n}\left(p_{0}\right) .
$$

But then, assuming that $\alpha \neq 0$, for every $F \in C^{\infty}(P)$, we have

$$
\begin{aligned}
\{F, H\}\left(p_{0}\right)+(F, S)\left(p_{0}\right)= & \left\langle\mathbf{d} F\left(p_{0}\right), \pi\left(p_{0}\right)\left(\mathbf{d} H\left(p_{0}\right)\right)\right\rangle+\left\langle\mathbf{d} F\left(p_{0}\right), \kappa\left(p_{0}\right)\left(\mathbf{d} S\left(p_{0}\right)\right)\right\rangle \\
= & \left\langle\mathbf{d} F\left(p_{0}\right), \frac{1}{\alpha} \pi\left(p_{0}\right)\left(\mathbf{d} S\left(p_{0}\right)-\beta_{1} \mathbf{d} C_{1}\left(p_{0}\right)-\cdots-\mathbf{d} C_{n}\left(p_{0}\right)\right)\right\rangle \\
& \quad+\left\langle\mathbf{d} F\left(p_{0}\right), \kappa\left(p_{0}\right)\left(\alpha \mathbf{d} H\left(p_{0}\right)+\beta_{1} \mathbf{d} C_{1}\left(p_{0}\right)+\cdots+\mathbf{d} C_{n}\left(p_{0}\right)\right)\right\rangle \\
= & \frac{1}{\alpha}\{F, S\}\left(p_{0}\right)-\frac{\beta_{1}}{\alpha}\left\{F, C_{1}\right\}\left(p_{0}\right)-\cdots-\frac{\beta_{n}}{\alpha}\left\{F, C_{n}\right\}\left(p_{0}\right) \\
& \quad+\alpha(F, H)\left(p_{0}\right)+\beta_{1}\left(F, C_{1}\right)\left(p_{0}\right)+\cdots+\beta_{n}\left(F, C_{n}\right)\left(p_{0}\right)=0
\end{aligned}
$$

which means that $p_{0}$ is an equilibrium of the metriplectic dynamics (36) or (37). This is akin to the free energy extremization of thermodynamics, as noted by Morrison [1984b] and Morrison [1986] where it was suggested that one can build in degeneracies associated with Hamiltonian "dynamical constraints." (See also Mielke [2011].)

Suppose that $K \in C^{\infty}(P)$ is a conserved quantity for the Hamiltonian part of the metriplectic dynamics, i.e., $\{K, H\}=$ 0 . Then, if $c(t)$ is an integral curve of the metriplectic dynamics, we have

$$
\begin{aligned}
\frac{d}{d t} K(c(t)) & =\mathbf{d} K(c(t))(\dot{c}(t))=\langle\mathbf{d} F(c(t)), \pi(c(t))(\mathbf{d} H(c(t)))\rangle+\langle\mathbf{d} F(c(t)), \kappa(c(t))(\mathbf{d} S(c(t)))\rangle \\
& =\{K, H\}(c(t))+(K, S)(c(t))=(K, S)(c(t)) .
\end{aligned}
$$

As pointed out in Morrison [1986], this immediately implies that a function that is simultaneously conserved for the full metriplectic dynamics and its Hamiltonian part, is necessarily conserved for the dissipative part. Physically, it is advantageous for general metriplectic systems to conserve dynamical constraints, i.e., conserved quantitates of its Hamiltonian part and the examples given in Kaufman [1984], Morrison [1984a], Morrison [1984b], and Morrison [1986] satisfy this condition.

\subsection{Metriplectic systems based on Lie algebra triple brackets}

Associated with any quadratic Lie algebra (i.e., a Lie algebra admitting a bilinear symmetric invariant form) is a natural completely antisymmetric triple bracket. This is used to construct Lie algebra based metriplectic systems. The algebra $\mathfrak{s o}(3)$ is worked out explicitly and examples are given. 


\subsubsection{General theory}

A quadratic Lie algebra is, by definition, a Lie algebra admitting a bilinear symmetric non-degenerate invariant form $\kappa: \mathfrak{g} \times \mathfrak{g} \rightarrow \mathbb{R}$ (the letter $\kappa$ is meant to remind one of the Killing form in a semisimple Lie algebra). Recall that invariance means that $\kappa([\xi, \eta], \zeta)=\kappa(\xi,[\eta, \zeta])$ for all $\xi, \eta, \zeta \in \mathfrak{g}$ or, equivalently, that the adjoint operators ad $\eta$ for all $\eta \in \mathfrak{g}$ are antisymmetric relative to $\kappa$. Non-degeneracy (strong) means that the map $\mathfrak{g} \ni \xi \mapsto \kappa(\xi, \cdot) \in \mathfrak{g}^{*}$ is an isomorphism. Finite dimensional quadratic Lie algebras have been completely classified in Medina and Revoy [1985]. For finite dimensional Lie algebras, non-degeneracy is equivalent to the following statement: $\kappa(\xi, \eta)=0$ for all $\eta \in \mathfrak{g}$ if and only if $\xi=0$. In infinite dimensions this condition is called weak non-degeneracy and it is implied by non-degeneracy but the converse is, in general, false.

For example, let $\mathfrak{g}$ be an arbitrary finite dimensional Lie algebra. Recall that the Killing form is defined by $\kappa(\xi, \eta):=$ $\operatorname{Trace}\left(\operatorname{ad}_{\xi} \circ \operatorname{ad}_{\eta}\right)$. If $\left\{e_{i}\right\}, i=1, \ldots \operatorname{dim} \mathfrak{g}$, is an arbitrary basis of $\mathfrak{g}$ and $c^{p}{ }_{i j}$ are the structure constants of $\mathfrak{g}$, i.e., $\left[e_{i}, e_{j}\right]=$ $c^{p}{ }_{i j} e_{p}$, then

$$
\kappa(\xi, \eta)=\xi^{i} c^{p}{ }_{i q} \eta^{j} c^{q}{ }_{j p}
$$

and hence the components of $\kappa$ in the basis $\left\{e_{i}\right\}, i=1, \ldots \operatorname{dim} \mathfrak{g}$, are given by

$$
\kappa_{i j}=\kappa\left(e_{i}, e_{j}\right)=c^{p}{ }_{i q} c^{q}{ }_{j p} .
$$

The Killing form is bilinear symmetric and invariant; it is non-degenerate if and only if $\mathfrak{g}$ is semisimple. Moreover, $-\kappa$ is a positive definite inner product if and only if the Lie algebra $\mathfrak{g}$ is compact (i.e., it is the Lie algebra of a compact Lie group).

In general, let $\kappa$ be a bilinear symmetric non-degenerate invariant form and define the completely antisymmetric covariant 3-tensor

$$
c(\xi, \eta, \zeta):=\kappa(\xi,[\eta, \zeta])=-c(\xi, \zeta, \eta)=-c(\eta, \xi, \zeta)=-c(\zeta, \eta, \xi) .
$$

In the coordinates given by the basis $\left\{e_{i}\right\}, i=1, \ldots \operatorname{dim} \mathfrak{g}$, the components of $c$ are

$$
c_{i j k}:=\kappa_{i m} c^{m}{ }_{j k}=-c_{i k j}=-c_{j i k}=-c_{k j i} .
$$

This construction immediately leads to the triple bracket introduced by Bialynicki-Birula and Morrison [1991] (see also Morrison [1998]), $\{\cdot, \cdot, \cdot\}: C^{\infty}(\mathfrak{g}) \times C^{\infty}(\mathfrak{g}) \times C^{\infty}(\mathfrak{g}) \rightarrow C^{\infty}(\mathfrak{g})$ defined by

$$
\{f, g, h\}(\xi):=c(\nabla f(\xi), \nabla g(\xi), \nabla h(\xi)):=\kappa(\nabla f(\xi),[\nabla g(\xi), \nabla h(\xi)]),
$$

where the gradient is taken relative to the non-degenerate bilinear form $\kappa$, i.e., for any $\xi \in \mathfrak{g}$ we have

$$
\kappa(\nabla f(\xi), \cdot):=\mathbf{d} f(\xi)
$$

or, in coordinates

$$
\nabla^{i} f(\xi)=\kappa^{i j} \frac{\partial f}{\partial \xi^{i}}
$$

where $\left[\kappa^{i j}\right]=\left[\kappa_{k l}\right]^{-1}$, i.e., $\kappa^{i j} \kappa_{j k}=\delta_{k}^{i}$. This triple bracket is trilinear over $\mathbb{R}$, completely antisymmetric, and satisfies the Leibniz rule in any of its variables. In coordinates it is given by

$$
\begin{aligned}
\{f, g, h\} & =c_{i j k} \nabla^{i} f \nabla^{j} g \nabla^{k} h=\kappa_{i m} c^{m}{ }_{j k} \kappa^{i p} \frac{\partial f}{\partial \xi^{p}} \kappa^{j q} \frac{\partial g}{\partial \xi^{q}} \kappa^{k r} \frac{\partial h}{\partial \xi^{r}}=c^{p}{ }_{j k} \kappa^{j q} \kappa^{k r} \frac{\partial f}{\partial \xi^{p}} \frac{\partial g}{\partial \xi^{q}} \frac{\partial h}{\partial \xi^{r}} \\
& =c^{p q r} \frac{\partial f}{\partial \xi^{p}} \frac{\partial g}{\partial \xi^{q}} \frac{\partial h}{\partial \xi^{r}},
\end{aligned}
$$

where $c^{p q r}$ are the components of the contravariant completely antisymmetric 3-tensor $\bar{c}$ associated to $c$ by raising its indices with the non-degenerate symmetric bilinear form $\kappa$, i.e., for any $\xi, \eta, \zeta \in \mathfrak{g}$, we have

$$
\bar{c}(\kappa(\xi, \cdot), \kappa(\eta, \cdot), \kappa(\gamma, \cdot)):=c(\xi, \eta, \zeta) .
$$


This construction extends the bracket due to Nambu [1973] to a Lie algebra setting. Nambu considered ordinary vectors in $\mathbb{R}^{3}$ and defined

$$
\{f, g, h\}_{\mathrm{Nambu}}(\Pi)=\nabla f(\Pi) \cdot(\nabla g(\Pi) \times \nabla h(\Pi)),
$$

where '' and ' $\times$ ' are the ordinary dot and cross products. Thus, the Nambu bracket is a special case of the triple bracket (40) in the case of $\mathfrak{g}=\mathfrak{s o}(3)$, whose the structure constants are the completely antisymmetric Levi-Civita symbol $\varepsilon_{i j k}$. Such 'modified rigid body brackets' were also described in Bloch and Marsden [1990], Holm and Marsden [1991], and Marsden and Ratiu [1999].

If $\mathfrak{g}$ is an arbitrary quadratic Lie algebra with bilinear symmetric non-degenerate invariant form $\kappa$, the quadratic function

$$
C_{2}(\xi):=\frac{1}{2} \kappa(\xi, \xi)
$$

is a Casimir function for the Lie-Poisson bracket on $\mathfrak{g}$, identified with $\mathfrak{g}^{*}$ via $\kappa$, i.e.,

$$
\{f, g\}_{ \pm}(\xi)= \pm \kappa(\xi,[\nabla f(\xi), \nabla g(\xi)])
$$

as an easy verification shows since $\nabla C_{2}(\xi)=\xi$. In view of [43), the following identity is obvious

$$
\{f, g\}_{+}=\left\{C_{2}, f, g\right\}
$$

(this was first pointed out in Bialynicki-Birula and Morrison [1991]). For example, if $\mathfrak{g}=\mathfrak{s o}(3)$, the (-)Lie-Poisson bracket

$$
\{f, g\}_{-}^{\mathfrak{s o}(3)}(\Pi)=-\left\{C_{2}, f, g\right\}_{\mathrm{Nambu}}(\Pi)=-\Pi \cdot(\nabla f(\Pi) \times \nabla g(\Pi))
$$

is the rigid body bracket, i.e., if $h(\Pi)=\frac{1}{2} \Pi \cdot \Omega$, where $\Pi_{i}=I_{i} \Omega_{i}, I_{i}>0, i=1,2,3$, and $I_{i}$ are the principal moments of inertia of the body, then Hamilton's equations $\frac{d}{d t} F(\Pi)=\{f, h\}_{-}^{\mathfrak{s o}(3)}(\Pi)$ are equivalent to Euler's equations $\dot{\Pi}=$ $\Pi \times \Omega$.

Note that given any two functions, $f, g \in C^{\infty}(\mathfrak{g})$, because the triple bracket satisfies the Leibniz identity in every factor, the map $C^{\infty}(\mathfrak{g}) \ni h \mapsto\{h, f, g\} \in C^{\infty}(\mathfrak{g})$ is a derivation and hence defines a vector field on $\mathfrak{g}$, denoted by $X_{f, g}: \mathfrak{g} \rightarrow \mathfrak{g}$, i.e.,

$$
\left\langle\mathbf{d} h(\xi), X_{f, g}(\xi)\right\rangle=\kappa\left(\nabla h(\xi), X_{f, g}(\xi)\right)=\{h, f, g\}(\xi) \quad \text { for all } \quad h \in C^{\infty}(\mathfrak{g}) .
$$

Note that $X_{f, f}=0$. Thus, for triple brackets, two functions define a vector field, analogous to the Hamiltonian vector field defined by a single function associated to a standard Poisson bracket.

From (40) we have the following result.

Proposition 3. The vector field $X_{f, g}$ on $\mathfrak{g}$ corresponding to the pair of functions $f, g$ is given by

$$
X_{f, g}(\xi)=[\nabla f(\xi), \nabla g(\xi)] .
$$

Triple brackets of the form (40) can be used to construct metriplectic systems on a quadratic Lie algebra $\mathfrak{g}$ in the following manner. Let $\kappa$ be the bilinear symmetric non-degenerate form on $\mathfrak{g}$ defining the quadratic structure and fix some $h \in C^{\infty}(\mathfrak{g})$. Define the symmetric bracket

$$
(f, g)_{h}^{\kappa}(\xi):=-\kappa\left(X_{h, f}(\xi), X_{h, g}(\xi)\right) .
$$

Assume that $-\kappa$ is a positive definite inner product. Then $(f, f) \geq 0$. Thus we have the manifold $\mathfrak{g}$ endowed with the Lie-Poisson bracket [43], the symmetric bracket 477, the Hamiltonian $h$, and for the entropy $S$ we take any Casimir function of the Lie-Poisson bracket. Then the conditions (i)-(iii) of 4.1 are all satisfied, because $(h, g)_{h}^{\kappa}=-\kappa\left(X_{h, h}, X_{h, g}\right)=-\kappa\left(0, X_{h, g}\right)=0$ for any $g \in C^{\infty}(\mathfrak{g})$. The equations of motion (36) are in this case given by

$$
\begin{aligned}
\frac{d}{d t} f(\xi) & =\kappa\left(\nabla f(\xi), \frac{d}{d t} \xi\right)=\{f, h\}_{ \pm}(\xi)+(f, S)(\xi)= \pm \kappa(\xi,[\nabla f(\xi), \nabla h(\xi)])-\kappa\left(X_{h, f}(\xi), X_{h, S}(\xi)\right) \\
& =\mp \kappa(\nabla f(\xi),[\xi, \nabla h(\xi)])-\kappa([\nabla h(\xi), \nabla f(\xi)],[\nabla h(\xi), \nabla S(\xi)])
\end{aligned}
$$

for any $f \in C^{\infty}(\mathfrak{g})$. 
This gives the equations of motion

$$
\dot{\xi}= \pm[\xi, \nabla h(\xi)]+[\nabla h(\xi),[\nabla h(\xi), \nabla S(\xi)]]
$$

Note that the flow corresponding to $S$ is a generalized double bracket flow. Observe also that this flow reduces to a double bracket flow and is tangent to an orbit of the group if $\nabla h(\xi)=\xi$. Indeed if $h=\frac{1}{2} \kappa(\xi, \xi)$ the symmetric bracket (47) reduces to the symmetric bracket induced from the normal metric.

\subsubsection{Special case of $\mathfrak{s o}(3)$}

If the quadratic Lie algebra is $\mathfrak{s o}(3)$, we identify it with $\mathbb{R}^{3}$ with the cross product as Lie bracket via the Lie algebra isomorphism $^{\wedge}: \mathbb{R}^{3} \rightarrow \mathfrak{s o}(3)$ given by $\hat{\mathbf{u} v}:=\mathbf{u} \times \mathbf{v}$ for all $\mathbf{u}, \mathbf{v} \in \mathbb{R}^{3}$. Since $\operatorname{Ad}_{A} \hat{\mathbf{u}}=\widehat{A \mathbf{u}}$, for any $A \in S O(3)$ and $\mathbf{u} \in \mathbb{R}^{3}$, we conclude that the usual inner product on $\mathbb{R}^{3}$ is an invariant inner product. In terms of elements of $\mathfrak{s o}(3)$ we have $\mathbf{u} \cdot \mathbf{v}=-\frac{1}{2}$ Trace $(\hat{\mathbf{u}} \hat{\mathbf{v}})$. We shall show below that the metriplectic structure on $\mathbb{R}^{3}$ is precisely the one given in Morrison [1986].

Recall that the Nambu bracket is given for $\mathfrak{s o}(3)$ by (41) and hence the symmetric bracket (47) has the form

$$
\begin{aligned}
\kappa(\{\Pi, h, f\},\{\Pi, h, g\}) & =\varepsilon^{i m n} \frac{\partial h}{\partial \Pi^{m}} \frac{\partial f}{\partial \Pi^{n}} \delta_{i j} \varepsilon^{j s t} \frac{\partial h}{\partial \Pi^{s}} \frac{\partial g}{\partial \Pi^{t}} \\
& =\varepsilon^{i m n} \varepsilon_{i}^{s t} \frac{\partial h}{\partial \Pi^{m}} \frac{\partial f}{\partial \Pi^{n}} \frac{\partial h}{\partial \Pi^{s}} \frac{\partial g}{\partial \Pi^{t}} \\
& =\|\nabla h\|^{2} \nabla g \cdot \nabla f-(\nabla f \cdot \nabla h)(\nabla g \cdot \nabla h)
\end{aligned}
$$

where in the third equality we have used the identity $\varepsilon^{i m n} \varepsilon_{i}^{s t}=\delta^{m s} \delta^{n t}-\delta^{m t} \delta^{n s}$. This coincides with Morrison, 1986 , equation (31)].

With the choice $S(\Pi)=\|\Pi\|^{2} / 2$ and the usual rigid body Hamiltonian, the equations of motion (48) are those for the relaxing rigid body given in Morrison [1986].

\section{Comments.}

- In three dimensions any Poisson bracket can be written as

$$
\{f, g\}=J^{i j} \frac{\partial f}{\partial \Pi^{i}} \frac{\partial g}{\partial \Pi^{j}}=\varepsilon^{i j}{ }_{k} V^{k}(\Pi) \frac{\partial f}{\partial \Pi^{i}} \frac{\partial g}{\partial \Pi^{j}}
$$

where $i, j, k=1,2,3$, and $V \in \mathbb{R}^{3}$. The last equality follows from the identification of $3 \times 3$ antisymmetric matrices with vectors (the hat map discussed above). Using the well know fact (which is easy to show directly) that brackets of the form of (50) satisfy the Jacobi identity if

$$
V \cdot \nabla \times V=0
$$

we conclude that

$$
\{F, G\}_{f}=\{f, F, G\}_{\mathrm{Nambu}}
$$

satisfies the Jacobi identity for any smooth function $f$; i.e., unlike the general case where the theorem of Bialynicki-Birula and Morriso [1991] requires $f$ to be the quadratic Casimir, one obtains a good Poisson bracket for any $f$. Thus, for the special case of three dimensions, one can interchange the roles of Hamiltonian and entropy in the metriplectic formalism.

- Thinking in terms of $\mathfrak{s o}(3)^{*}$, the setting arising from reduction (see e.g. Marsden and Ratiu [1999]), this construction leads to a natural geometric interpretation of a metriplectic system on the manifold $P=\mathbb{R}^{3}$. With the Poisson bracket on $\mathbb{R}^{3}$ of $(52)$, the bundle map $\pi: T^{*} \mathbb{R}^{3} \rightarrow T \mathbb{R}^{3}$ has the expression

$$
\pi_{f}(x, \Pi)=\left(x, \nabla f(\Pi) \times(\cdot)^{\top}\right)
$$


since $\mathbf{d} H(\Pi)^{\top}=\nabla H(\Pi)(\mathbf{d} H(\Pi)$ is a row vector and $\nabla H(\Pi)$ is its transpose, a column vector). Now the triple bracket associated to the equation (48) can be used to generate a symmetric bracket given in Bloch. Krishnaprasad. Marsden, and Ratiu [1994] as follows:

$$
\begin{aligned}
(F, G)_{B K M R}(\Pi) & =(F, G)_{C}^{\kappa}=\kappa(\{\Pi, C, F\},\{\Pi, C, G\}) \\
& =(\Pi \times \nabla F(\Pi)) \cdot(\Pi \times \nabla G(\Pi)) .
\end{aligned}
$$

where now $C=\|\Pi\|^{2} / 2$. Hence the bundle map $\kappa: T^{*} \mathbb{R}^{3} \rightarrow T \mathbb{R}^{3}$ has the expression

$$
\kappa(x, \Pi)=-\Pi \times\left(\Pi \times(\cdot)^{\top}\right) .
$$

Thus, with the freedom to choose any quantity $S=f$ as an entropy, with the assurance that 51] will be satisfied because $\nabla \times V=\nabla \times \nabla f=0$, we can take $H=C$ and have $\{F, S\}_{f}=0$ and $(F, H)=0$ for all $F \in C^{\infty}\left(\mathbb{R}^{3}\right)$. The equations of motion for this metriplectic system are

$$
\dot{\Pi}=-\Pi \times \nabla f(\Pi)-\Pi \times(\Pi \times \nabla f(\Pi)) .
$$

The symmetric bracket is the inner product of the two Hamiltonian vector fields on each concentric sphere. As discussed in Bloch, Krishnaprasad, Marsden, and Ratiu [1994], this symmetric bracket can be defined on any compact Lie algebra by taking the normal metric on each coadjoint orbit.

- The following set of equations were given in Fish [2005]:

$$
\dot{\Pi}=\nabla S(\Pi) \times \nabla H(\Pi)-\nabla H(\Pi) \times(\nabla H(\Pi) \times \nabla S(\Pi)) .
$$

Yet, this metriplectic system is identical to that obtained from (48), using (49), viz.

$$
\dot{\Pi}=\{\Pi, S, H\}+\kappa(\{\Pi, H, \Pi\},\{\Pi, H, S\}),
$$

Replacing $H$ by $g$ in (49) gives

$$
(F, G)_{g}((\Pi))=\kappa(\{(\Pi), g, F\},\{(\Pi), g, G\})=(\nabla g(\Pi) \times \nabla F(\Pi)) \cdot(\nabla g(\Pi) \times \nabla G(\Pi)) .
$$

Thus, the bundle map $\kappa: T^{*} \mathbb{R}^{3} \rightarrow T \mathbb{R}^{3}$ has the expression

$$
\kappa_{g}(x, \Pi)=-\nabla g(\Pi) \times\left(\nabla \Pi \times(\cdot)^{\top}\right) .
$$

Examples: Two special cases of the equation (55) are of interest.

(i) If we take $H=\frac{1}{2}\|\Pi\|^{2}$ and $S=c \cdot \Pi, c$ a constant vector, we obtain

$$
\dot{\Pi}=c \times \Pi-\Pi \times(\Pi \times c) .
$$

(ii) If we take $S=\frac{1}{2}\|\Pi\|^{2}$ and $H=c \cdot \Pi, c$ a constant, we obtain

$$
\dot{\Pi}=\Pi \times c-c \times(c \times \Pi) .
$$

The equations of motion (58) is an instance of double bracket damping, where the damping is due to the normal metric, whereas (59) gives linear damping of the sort arising in quantum systems. 


\subsection{The Toda system revisited}

\subsubsection{The Toda lattice equation revisited}

We note that the Toda lattice equation fits into the metriplectic picture in a degenerate but interesting fashion since it has a dual Hamiltonian and gradient character which may be seen by writing it in the double bracket form (2). .

It may be viewed either as the Hamiltonian part or the dissipative part of a metriplectic system with Hamiltonian $H=\frac{1}{2} \operatorname{Tr} L^{2}$ or entropy function $S=\operatorname{Tr} L N$ respectively with the Toda lattice equations in the corresponding form (7) or (2), as discussed in Section 2 This observation may be extended to the Toda lattice flow on the normal form of any complex semisimple Lie algebra as can be see in Bloch, Brockett, and Ratiu [1992].

\subsubsection{Full Toda with dissipation}

It is possible to construct an interesting metriplectic system which incorporates the full Toda dynamics.

We consider the again the flow on the vector space of symmetric matrices $\mathfrak{k}^{\perp}=\mathfrak{s y \mathfrak { m }}(n)$ but now consider the flow on a generic orbit as discussed in Deift et al. [1992] where it was shown that the flow is integrable. The Hamiltonian is again $\frac{1}{2} \operatorname{Tr} L^{2}$ and the flow on full symmetric matrices is given by

$$
\dot{L}=\left[\pi_{\mathfrak{s}} L, L\right]
$$

with $\pi_{\mathfrak{s}}$ being the projection onto the skew symmetric matrices in the lower triangular skew decomposition of a matrix. In this setting there are nontrivial Casimir functions of the bracket (9). These are given as follows. For $L$ an $n \times n$ symmetric matrix set for $0 \leq k \leq\left[\frac{1}{2} n\right]$

$$
\operatorname{det}(L-\lambda)_{k}=\sum_{r-0}^{n-2 k} E_{r k}(L) \lambda^{n-2 k-r}
$$

where the subscript $k$ denotes the matrix obtained by deleting the first $k$ rows and the last $k$ columns. Then $I_{1 k}(L)=$ $E_{1 k}(L) / E_{0 k}(L)$ are Casimir functions of the generic orbit in $\mathfrak{s y m}(n)$ as shown in Deift et al. [1992].

Thus we obtain the metriplectic systems

$$
\dot{L}=\left[\pi_{\mathfrak{s}} L, L\right]+\left[L,\left[L, \nabla I_{1 k}\right]\right]
$$

where the metric is the normal metric on orbits of $\mathfrak{s u}(n)$ restricted to the symmetric matrices (identified with $i$ times the symmetric matrices) as in Bloch. Brockett, and Ratiu [1992]. Here $H=\frac{1}{2} \operatorname{Tr} L^{2}$ and $S=I_{1 k}$.

\subsection{Metriplectic systems for pdes: metriplectic brackets and examples}

First we construct a class of metriplectic brackets based on triple brackets for infinite systems, then we consider in detail an example based on Gardner's bracket on $S^{1}$. Lastly, we mention various generalizations.

\subsubsection{Symmetric brackets for pdes based on triple brackets}

Similar to 4.2 we can construct metriplectic flows for infinite-dimensional systems from completely antisymmetric triple brackets of the form

$$
\{E, F, G\}=\int_{S^{1}} \mathrm{~d} \theta_{1} \int_{S^{1}} \mathrm{~d} \theta_{2} \int_{S^{1}} \mathrm{~d} \theta_{3} \mathscr{C}_{i j k}\left(\theta_{1}, \theta_{2}, \theta_{3}\right)\left(\mathscr{P}^{i} E_{u}\right)\left(\theta_{1}\right)\left(\mathscr{P}^{j} F_{u}\right)\left(\theta_{2}\right)\left(\mathscr{P}^{k} G_{u}\right)\left(\theta_{3}\right)
$$

where $E, F$, and $G$ are smooth functions on $S^{1}, \mathscr{C}_{i j k}$ is a smooth function on $S^{1} \times S^{1} \times S^{1}$ which is completely antisymmetric in its arguments, so as to assure complete antisymmetry of $\{E, F, G\}$. In addition, we denote $E_{u}:=\delta E / \delta u$, 
etc. Let $\mathscr{P}^{i}, i=1,2,3$, be pseudo-differential operators. Evidently, the triple bracket of 63 is trilinear and completely antisymmetric in $E, F, G$.

From 63 and a Hamiltonian $H$, we construct a symmetric bracket as follows:

$$
(F, G)_{H}=\int_{S^{1}} \mathrm{~d} \theta^{\prime} \int_{S^{1}} \mathrm{~d} \theta^{\prime \prime}\left\{U\left(\theta^{\prime}\right), H, F\right\} \mathscr{G}\left(\theta^{\prime}, \theta^{\prime \prime}\right)\left\{U\left(\theta^{\prime \prime}\right), H, G\right\},
$$

where $U(\theta)$ in 64 denotes the functional

$$
U(\theta): u \mapsto \int_{S^{1}} \mathrm{~d} \theta^{\prime} u\left(\theta^{\prime}\right) \delta\left(\theta-\theta^{\prime}\right)
$$

We shall use this notation in subsequent expressions below. The 'metric' $\mathscr{G}$ is assumed to be symmetric and positive semidefinite, i.e., the smooth function $\mathscr{G}: S^{1} \times S^{1} \rightarrow \mathbb{R}$ satisfies $\mathscr{G}\left(\theta^{\prime}, \theta^{\prime \prime}\right)=\mathscr{G}\left(\theta^{\prime \prime}, \theta^{\prime}\right)$ and

$$
\int_{S^{1}} \mathrm{~d} \theta^{\prime} \int_{S^{1}} \mathrm{~d} \theta^{\prime \prime} \mathscr{G}\left(\theta^{\prime}, \theta^{\prime \prime}\right) f\left(\theta^{\prime}\right) f\left(\theta^{\prime \prime}\right) \geq 0
$$

for all functions $f \in C^{\infty}\left(S^{1}\right)$. Therefore, by construction, it is clear that 64 satisfies the following:

(i) $(F, G)_{H}=(G, F)_{H}$ for all $F, G$,

(ii) $(F, H)_{H}=0$ for all $F$, and

(iii) $(F, F)_{H} \geq 0$ for all $F$.

As a special case suppose $\mathscr{P}^{i}=\mathscr{P}$ for all $i=1,2,3$; then becomes

$$
\{E, F, G\}=\int_{S^{1}} \mathrm{~d} \theta_{1} \int_{S^{1}} \mathrm{~d} \theta_{2} \int_{S^{1}} \mathrm{~d} \theta_{3} \mathscr{C}\left(\theta_{1}, \theta_{2}, \theta_{3}\right) \mathscr{P}\left(\theta_{1}\right) E_{u} \mathscr{P}\left(\theta_{2}\right) F_{u} \mathscr{P}\left(\theta_{3}\right) G_{u} .
$$

As a further specialization, suppose $\mathscr{C}\left(\theta_{1}, \theta_{2}, \theta_{3}\right)$ is given by

$$
\mathscr{C}\left(\theta_{1}, \theta_{2}, \theta_{3}\right)=A\left(\theta_{1}, \theta_{2}\right)+A\left(\theta_{2}, \theta_{3}\right)+A\left(\theta_{3}, \theta_{1}\right)
$$

where $A$ is any antisymmetric function, i.e.,

$$
A\left(\theta_{1}, \theta_{2}\right)=-A\left(\theta_{2}, \theta_{1}\right)
$$

The form (68), assuming 69, assures complete antisymmetry of $\mathscr{C}$.

Finally, a particularly interesting, self-contained, case would be to suppose the $A$ 's come from some Poisson bracket, according to

$$
A\left(\theta_{1}, \theta_{2}\right)=\left\{U\left(\theta_{1}\right), U\left(\theta_{2}\right)\right\} .
$$

It would be quite natural to choose the entropy, $S$, to be a Casimir function of this bracket and to choose this bracket as the Hamiltonian part of the metriplectic system with symmetric bracket given by (64). We give an example of this construction in Sec. 4.4.2

It is evident that one can construct a wide variety of symmetric brackets based on triple brackets. For example, one can choose the pseudo-differential operators from the list $\left\{\mathscr{I}_{d}, d / d \theta,(d / d \theta)^{-1}, \mathscr{H}\right\}$, where $\mathscr{I}_{d}$ is the identity operator, and the Hamiltonian, $H$, and entropy (Casimir) $C$ could be one of the following functionals:

$$
\begin{aligned}
H_{0} & =\int_{S^{1}} \mathrm{~d} \theta u \\
H_{2} & =\int_{S^{1}} \mathrm{~d} \theta u^{2} / 2 \\
H_{1} & =\int_{S^{1}} \mathrm{~d} \theta u^{\prime 2} / 2 \\
H_{K d V} & =\int_{S^{1}} \mathrm{~d} \theta\left(u^{3}+u^{\prime 2} / 2\right) .
\end{aligned}
$$


In the Sec. 4.4.2 we will construct a metriplectic system based on the Gardner bracket (30) of Sec. 3.7 To avoid complications, we choose a simple example, yet one that displays general features of a large class of $1+1$ energy conserving dissipative system.

\subsubsection{Metriplectic systems based on the Gardner bracket}

For simplicity we choose $\mathscr{P}_{i}=\mathscr{I}_{d}$ for all $i$, and as mentioned above, we suppose $A\left(\theta_{1}, \theta_{2}\right)$ is generated from the Gardner bracket $\underline{30}$, i.e.,

$$
A\left(\theta_{1}, \theta_{2}\right):=\left\{U\left(\theta_{1}\right), U\left(\theta_{2}\right)\right\}=\int_{S^{1}} d \theta \delta\left(\theta-\theta_{1}\right) \frac{d}{d \theta} \delta\left(\theta-\theta_{2}\right)=\delta^{\prime}\left(\theta_{1}-\theta_{2}\right),
$$

where prime denotes differentiation with respect to argument and $\delta^{\prime}\left(\theta_{1}-\theta_{2}\right)$ is defined by

$$
\begin{aligned}
\int_{S^{1}} \mathrm{~d} \theta_{1} \int_{S^{1}} \mathrm{~d} \theta_{2} \delta^{\prime}\left(\theta_{1}-\theta_{2}\right) f\left(\theta_{1}\right) g\left(\theta_{2}\right) & =-\int_{S^{1}} \mathrm{~d} \theta_{1} \int_{S^{1}} \mathrm{~d} s \delta^{\prime}(s) f\left(\theta_{1}\right) g\left(\theta_{1}-s\right)=\int_{S^{1}} \mathrm{~d} \theta_{1} f\left(\theta_{1}\right) g^{\prime}\left(\theta_{1}\right) \\
& =-\int_{S^{1}} \mathrm{~d} \theta_{1} \int_{S^{1}} \mathrm{~d} \theta_{2} \delta^{\prime}\left(\theta_{2}-\theta_{1}\right) f\left(\theta_{1}\right) g\left(\theta_{2}\right)
\end{aligned}
$$

for any $f, g \in C^{\infty}\left(S^{1}\right)$, which shows that $\delta^{\prime}\left(\theta_{2}-\theta_{1}\right)=-\delta^{\prime}\left(\theta_{1}-\theta_{2}\right)$. With this choice for $A$ we obtain

$$
\mathscr{C}\left(\theta_{1}, \theta_{2}, \theta_{3}\right)=\delta^{\prime}\left(\theta_{1}-\theta_{2}\right)+\delta^{\prime}\left(\theta_{2}-\theta_{3}\right)+\delta^{\prime}\left(\theta_{3}-\theta_{1}\right),
$$

and Eq. 67] becomes

$$
\begin{aligned}
\{E, F, G\}= & \int_{S^{1}} \mathrm{~d} \theta_{1} \int_{S^{1}} \mathrm{~d} \theta_{2} \int_{S^{1}} \mathrm{~d} \theta_{3}\left[\delta^{\prime}\left(\theta_{1}-\theta_{2}\right)+\delta^{\prime}\left(\theta_{2}-\theta_{3}\right)+\delta^{\prime}\left(\theta_{3}-\theta_{1}\right)\right] E_{u}\left(\theta_{1}\right) F_{u}\left(\theta_{2}\right) G_{u}\left(\theta_{3}\right) \\
= & \left(\int_{S^{1}} \mathrm{~d} \bar{\theta} G_{u}(\bar{\theta})\right) \int_{S^{1}} \mathrm{~d} \theta F_{u}(\theta) E_{u}^{\prime}(\theta)+\left(\int_{S^{1}} \mathrm{~d} \bar{\theta} E_{u}(\bar{\theta})\right) \int_{S^{1}} \mathrm{~d} \theta G_{u}(\theta) F_{u}^{\prime}(\theta) \\
& +\left(\int_{S^{1}} \mathrm{~d} \bar{\theta} F_{u}(\bar{\theta})\right) \int_{S^{1}} \mathrm{~d} \theta E_{u}(\theta) G_{u}^{\prime}(\theta) .
\end{aligned}
$$

We shall construct a metriplectic system of the form

$$
\dot{F}=\{H, F, G\}+\int_{S^{1}} \mathrm{~d} \theta^{\prime} \int_{S^{1}} \mathrm{~d} \theta^{\prime \prime}\left\{U\left(\theta^{\prime}\right), S, F\right\} \mathscr{G}\left(\theta^{\prime}, \theta^{\prime \prime}\right)\left\{U\left(\theta^{\prime \prime}\right), S, G\right\},
$$

using the Gardner bracket (71).

Observe if we now set $F=H_{0}$, the Casimir for the Gardner bracket (71), then, since $\delta H_{0} / \delta u=1$, we obtain

$$
\left\{F, H_{0}, G\right\}=\int_{S^{1}} \mathrm{~d} \theta F_{u} G_{u}^{\prime}
$$

which is precisely the Gardner bracket. To see this, let us compute, for example, the integral in the third term of (76). Changing variables $s=\theta_{3}-\theta_{1}$ we get

$$
\left.\int_{S^{1}} \mathrm{~d} \theta_{1} \int_{S^{1}} \mathrm{~d} \theta_{2} \int_{S^{1}} \mathrm{~d} \theta_{3} \delta^{\prime}\left(\theta_{3}-\theta_{1}\right) E_{u}\left(\theta_{1}\right) G_{u}\left(\theta_{3}\right)=-\int_{S^{1}} \mathrm{~d} s \int_{S^{1}} \mathrm{~d} \theta_{3} \delta^{\prime}(s) E_{u}\left(\theta_{3}-s\right) G_{u}\left(\theta_{3}\right)=\int_{S^{1}} \mathrm{~d} \theta_{3} E_{u}^{\prime}\left(\theta_{3}\right) G_{u} \theta_{3}\right) .
$$

A similar computation shows that the first and second terms vanish.

In order to construct the symmetric bracket in (64), we need the following, computed using (76):

$$
\{U(\theta), H, G\}=-\left(\int_{S^{1}} \mathrm{~d} \bar{\theta} G_{u}(\bar{\theta})\right) H_{u}^{\prime}(\theta)+\int_{S^{1}} \mathrm{~d} \bar{\theta} G_{u}(\bar{\theta}) H_{u}^{\prime}(\bar{\theta})+\left(\int_{S^{1}} \mathrm{~d} \bar{\theta} H_{u}(\bar{\theta})\right) G_{u}^{\prime}(\theta) .
$$

Now with the counterpart of 78 for the functional $F$ with $U\left(\theta^{\prime}\right)$, a choice for $H$, and a choice for $\mathscr{G}$, we can construct $(F, G)_{H}$. We make the following choices: 


$$
\begin{aligned}
& H_{2}(u)=\int_{S^{1}} \mathrm{~d} \theta \frac{u^{2}}{2}, \quad S(u):=H_{0}(u)=\int_{S^{1}} \mathrm{~d} \theta u \\
& \mathscr{G}\left(\theta^{\prime}, \theta^{\prime \prime}\right)=\delta\left(\theta^{\prime}-\theta^{\prime \prime}\right) .
\end{aligned}
$$

Now choose $\mathrm{H}_{2}$ from (79) and insert it into (78) which gives

$$
\left\{U(\theta), H_{2}, G\right\}=-\left(\int_{S^{1}} \mathrm{~d} \bar{\theta} G_{u}(\bar{\theta})\right) u^{\prime}(\theta)+\int_{S^{1}} \mathrm{~d} \bar{\theta} G_{u}(\bar{\theta}) u^{\prime}(\bar{\theta})+S G_{u}^{\prime}(\theta)
$$

and to construct the symmetric bracket (64), we need

$$
\left\{U\left(\theta^{\prime \prime}\right), H_{2}, S\right\}=-u^{\prime}\left(\theta^{\prime \prime}\right) .
$$

Thus, the equations of motion are

$$
\frac{d}{d t} F=\left\{F, H_{0}, H_{2}\right\}+(F, S)_{H_{2}}
$$

where

$$
(F, S)_{H_{2}}=\int_{S^{1}} \mathrm{~d} \theta^{\prime} \int_{S^{1}} \mathrm{~d} \theta^{\prime \prime}\left\{U\left(\theta^{\prime}\right), H_{2}, F\right\} \mathscr{G}\left(\theta^{\prime}, \theta^{\prime \prime}\right)\left\{U\left(\theta^{\prime \prime}\right), H_{2}, S\right\} .
$$

This yields

$$
u_{t}-u_{\theta}=S u_{\theta \theta}+Q \quad \text { with } \quad Q:=\int_{S^{1}} \mathrm{~d} \theta^{\prime}\left|u_{\theta^{\prime}}\right|^{2} .
$$

Equation (84) has several interesting features. For fixed given constant $S$ and $Q$, it is a linear equation composed of the heat equation with a source and with the inclusion of a linear advection term. One can proceed to solve this equation by the usual method of constructing a temporal Green's function out of the heat kernel and expanding in a Fourier series. After such a solution is constructed, one must enforce the fact that the global quantities $S$ and $Q$ are both time dependent and, importantly, dependent on the solution so constructed. Only after these constraints are enforced would one actually have a solution. Pursuing this construction, although interesting, is outside the scope of the present paper and will be treated elsewhere.

We observe that the equation (84) is metriplectic. Indeed, by construction, we have a Poisson bracket (77) (the Gardner bracket) and a symmetric bracket (83). Since these were constructed out of triple brackets, property (iii) of Definition in Section 4.1 holds. Positive semidefiniteness of the symmetric bracket follows from [81].

The nature of the dissipation of 84 is of particular interest in that it involves the global quantities $S$ and $Q$. This is reminiscent of collision operators, such as that due to Boltzmann and generalized nonlinear Fokker-Planck operators such as those due to Landau, Lenard-Balescu, and others (see, e.g., Morrison [1986]). The usual dissipation in $1+1$ systems is local in nature (see Sec. 4.5) and dissipates energy. Thus the metriplectic construction of this section has pointed to a quite natural type of dynamical system that has dynamical versions of both the first and second laws of thermodynamics. The pathway for constructing other systems with nonlinear and dispersive Hamiltonian components, other kinds of dissipation, etc. is now cleared, and some will be considered in future publications.

\subsubsection{Some metriplectic generalizations}

It is evident that many generalizations are possible. We mention a few.

- Without destroying the symmetries or formal metriplectic bracket properties we could allow one or both of the functions $C$ and $\mathscr{G}$ to depend on the field variable $u$ or even contain pseudodifferential operations. In fact, such ideas were used in similar brackets in Flierl and Morrison [2011] to facilitate numerical computation.

- It is clear how to generalize (64) to preserve more constraints, say $I_{1}, I_{2}, \ldots$, in addition to $H$. One simply first constructs the completely antisymmetric multilinear brackets $\{E, F, G, H, \ldots\}$ paralleling [63), and then, analogous to (64), constructs

$$
(F, G)_{H, I_{1}, I_{2}, \ldots}=\int_{S^{1}} \mathrm{~d} \theta^{\prime} \int_{S^{1}} \mathrm{~d} \theta^{\prime \prime}\left\{U\left(\theta^{\prime}\right), H, I_{1}, I_{2}, \ldots, F\right\} \mathscr{G}\left(\theta^{\prime}, \theta^{\prime \prime}\right)\left\{U\left(\theta^{\prime \prime}\right), H, I_{1}, I_{2}, \ldots, G\right\} .
$$

The bracket $(F, G)_{H, I_{1}, I_{2}, \ldots}$ is guaranteed to be symmetric, conserve the invariants, and be positive semidefinite. 
- It is of general interest to have metriplectic systems of the form

$$
\dot{F}=\{H, F, G\}+\int_{S^{1}} \mathrm{~d} \theta^{\prime} \int_{S^{1}} \mathrm{~d} \theta^{\prime \prime}\left\{U\left(\theta^{\prime}\right), S, F\right\} \mathscr{G}\left(\theta^{\prime}, \theta^{\prime \prime}\right)\left\{U\left(\theta^{\prime \prime}\right), S, G\right\}
$$

(such as our example of Sec.4.4.2) for a suitably chosen function $G$; here $H$ is the Hamiltonian and $S$ is the entropy. Exploring the mathematics of when this is possible is an area to pursue.

- The construction here is easily extendable to higher spatial dimensions. For example consider the following triple bracket given in Bialynicki-Birula and Morrison [1991]:

$$
\{E, F, G\}=\int_{\mathscr{D}} d^{6} z E_{f}\left[F_{f}, G_{f}\right],
$$

where $z=(q, p)$ is a canonical six-dimensional phase space variable, $f(z, t)$ is a phase space density, as in Vlasov theory, the 'inner' Poisson bracket is defined by

$$
[f, g]=f_{q} \cdot g_{p}-f_{p} \cdot g_{q}
$$

We assume that the domain $\mathscr{D}$ with boundary conditions enables us to set all surface terms obtained by integrations by parts to zero, thereby assuring complete antisymmetry. Inserting the quadratic Casimir $C_{2}:=\int_{\mathscr{D}} d^{6} z f^{2} / 2$ into 86 gives

$$
\{F, G\}_{V P}=\left\{C_{2}, F, G\right\}=\int_{\mathscr{D}} d^{6} z f\left[F_{f}, G_{f}\right],
$$

the Lie-Poisson bracket for the Vlasov-Poisson system, as given in Morrison [1980]. Thus, this bracket with the quadratic Casimir is formally akin to the construction given in Sec. 4.2.1 (although we note it reduces to a good bracket for any Casimir and in this way is like the case of $\mathfrak{s o}(3)$ of Sec.4.2.2. The triple bracket of (86) can be used in a generalization of the bracket of (64) to obtain a variety of energy conserving collision operators, with a wide choice of Casimirs as entropies.

\subsection{Hybrid dissipative structures}

Even if a system is not metriplectic, it is of interest to see if it can be obtained from an equation which consists of a Hamiltonian part and a gradient part with respect to a suitable Poisson bracket and metric, respectively.

For KdV-like equations, energy (the Hamiltonian) is generally not conserved when dissipation is added to the system. This is common for physical systems, but a more complete model would conserve energy while accounting for heat loss, i.e., entropy production. In the terminology of Morrison [2009], models that lose energy, such as those treated here and those described by the double bracket formalism of 2.1 are incomplete, while those that do represent dynamical models of the laws of thermodynamics, such as metriplectic systems, are termed complete. Although incomplete systems do not conserve energy, they may conserve other invariants, and building this in, represents an advantage of various bracket formulations. Thus, we construct incomplete hybrid Hamiltonian and dissipative dynamics by combining a Hamiltonian and a gradient vector field according to the prescription

$$
u_{t}=\{u, H\}+(u, S)
$$

where $u \mapsto\{u, H\}$ is a Hamiltonian vector field generated by $H$ and $u \mapsto(u, S)$ is a gradient vector field generated by $S$ (which could be $H$ ). Thus, (,) is, up to a sign, an inner product on the space of functions $u$.

Consider the following examples:

- With the usual KdV Hamiltonian of (31) and the Gardner bracket of (30) describing the Hamiltonian vector field, together with the choice

$$
S(u)=H_{1}(u)=\frac{1}{4 \pi} \int_{-\pi}^{\pi} \mathrm{d} \theta\left(u_{\theta}\right)^{2}
$$

we obtain for the gradients of Corollary 2 
(i) $u_{t}=\{u, H\}-\nabla^{1} H_{1}=-u_{\theta \theta \theta}+6 u u_{\theta}-u$

(ii) $u_{t}=\{u, H\}-\nabla H_{1}=-u_{\theta \theta \theta}+6 u u_{\theta}+u_{\theta \theta}$

(iii) $u_{t}=\{u, H\}-\nabla^{2} H_{1}=-u_{\theta \theta \theta}+6 u u_{\theta}-\mathscr{H}\left(u_{\theta}\right)$

which is the $\mathrm{KdV}$ equation of (29) with the inclusion of a new term that describes dissipation. Case (i) corresponds to simple linear damping, case (ii) to 'viscous' diffusion, and case (iii) to the equation of Ott and Sudan [1969] which adds a term to the $\mathrm{KdV}$ equation that describes Landau damping. For these systems the $\mathrm{KdV}$ invariant $\int_{-\pi}^{\pi} \mathrm{d} \theta u^{2}$ serves as a Lyapunov function.

- Choosing $H=S=H_{1}$, the Kähler Hamiltonian flow of (34) together with the dissipative flow generated by (21), yields

$$
u_{t}=\left\{u, H_{1}\right\}-\nabla^{2} H_{1}=-u_{\theta}-\mathscr{H}\left(u_{\theta}\right)
$$

which describes simple advection with Landau damping. This equation possesses the damped traveling wave solution.

- We note that we can derive the heat equation from a symmetric bracket of the form (64), again with $\mathscr{G}\left(\theta^{\prime}, \theta^{\prime \prime}\right)=$ $\delta\left(\theta^{\prime}-\theta^{\prime \prime}\right)$. Using this $\mathscr{G}$ and noting $\left\{U(\theta), H_{0}, F\right\}=G_{u}^{\prime}(\theta)$, we obtain

$$
(F, G)_{H_{0}}=\int_{S^{1}} d \theta F_{u}^{\prime} G_{u}^{\prime} .
$$

Let us compute, for example, $\dot{F}(u)=\left(F,-H_{2}\right)_{H_{0}}$ (see (72). Since $\delta H_{2} / \delta u=-u$, we obtain

$$
\int_{S^{1}} \mathrm{~d} \theta F_{u} \dot{u}=\frac{d}{d t} F(u)=\left(F, H_{2}\right)_{H_{0}}=-\int_{S^{1}} \mathrm{~d} \theta F_{u}^{\prime} u^{\prime}=\int_{S^{1}} \mathrm{~d} \theta F_{u} u^{\prime \prime} .
$$

This yields

$$
u_{t}=u_{x x}
$$

which is the heat equation.

From these examples it is clear how a variety of hybrid Hamiltonian and dissipative flows can be constructed from the machinery we have developed. For example, if we replace the KdV Hamiltonian by $H(u)=\int_{S^{1}} \mathrm{~d} \theta\left(\frac{1}{2} u \mathscr{H}\left(u_{\theta}\right)+\frac{1}{3} u^{3}\right)$ we obtain the Benjamin-Ono equation with the various dissipative terms. Related ideas apply to fluid dynamics may be found in Gay-Balmaz and Holm [2012].

Acknowledgements AMB was partially supported by NSF grants DMS-090656 and DMS-1207693. PJM was supported by U.S. Department of Energy contract DE-FG05-80ET-53088. TSR was partially supported by Swiss NSF grant 200021-140238, and by the government grant of the Russian Federation for support of research projects implemented by leading scientists, Lomonosov Moscow State University under the agreement No. 11.G34.31.0054.

\section{References}

Abraham, R. and J. E. Marsden [1978], Foundations of Mechanics, Second edition, Addison-Wesley, Reprinted by Perseus Press, 1997.

Adams, R. A. and J. J. F. Fournier [2003], Sobolev Spaces, Second edition. Pure and Applied Mathematics, 140, Elsevier/Academic Press, Amsterdam.

Adler, M. [1979], On a trace functional for formal pseudo differential operators and the symplectic structure of the Korteweg-de Vries type equations, Invent. Math. 50(3), 219-248.

Atiyah, M. F. [1982] Convexity and commuting Hamiltonians, Bull. London Math. Soc. 14, 305-315.

Atiyah, M. F. and A. N. Pressley [1983], Convexity and loop groups, in Arithmetic and Geometry, Vol. II, 33-63, Progress in Mathematics, 36, Birkhäuser Boston, Boston, MA, 1983.

Beltita, D. [2005], Integrability of analytic almost complex structures on Banach manifolds, Annals of Global Anal. and Geom. 28, 59-73.

Besse, A. L. [2008], Einstein Manifolds, Reprint of the 1987 edition. Classics in Mathematics. Springer-Verlag, Berlin. 
Bialynicki-Birula, I. and P. J. Morrison [1991], Quantum mechanics as a generalization of Nambu dynamics to the Weyl-Wigner formalism, Phy. Lett. A 158, 453-457.

Bloch A. M. [2003], Nonholonomic Mechanics and Control, Springer, New York.

Bloch, A. M. [1990], Steepest descent, linear programming and Hamiltonian flows, Contemp. Math. AMS 114, 77-88.

Bloch, A. M., R. W. Brockett and T. S. Ratiu [1990], A new formulation of the generalized Toda Lattice equations and their fixed point analysis via the momentum map, Bull. Amer. Math. Soc. 23, 477-485.

Bloch, A. M., R. W. Brockett and T. S. Ratiu [1992], Completely integrable gradient flows, Comm. Math. Phys. 147, 57-74.

Bloch, A. M., H. Flaschka and T. S. Ratiu [1990], A convexity theorem for isospectral manifolds of Jacobi matrices in a compact Lie algebra, Duke Math. J. 61, 41-65.

Bloch, A. M. and A. Iserles [2005], Aspects of generalized double bracket flows, Proc. Centre de Recherche Montreal, $A M S$, Group Theory and Numerical Analysis 39, 65-76.

Bloch, A. M., P. S. Krishnaprasad, J. E. Marsden, and T. S. Ratiu [1994], Dissipation Induced Instabilities, Ann. Inst. H. Poincaré, Analyse Nonlineare 11, 37-90.

Bloch, A. M., P. S. Krishnaprasad, J. E. Marsden, and T. S. Ratiu [1996], The Euler-Poincaré equations and double bracket dissipation, Comm. Math. Phys. 175, 1-42.

Bloch, A. M. and J. E. Marsden [1990], Stabilization of rigid body dynamics by the energy-Casimir method, Systems and Control Letters 14, 341-346.

Bourbaki, N. [1971], Lie Groups and Lie Algebras. Chapters 1-3, Springer-Verlag, Berlin, 1998. Translated from the 1971 French edition.

Brockett, R. [1991], Dynamical systems that sort lists, solve linear programming problems and diagonalize symmetric matrices, Proc. 1988 IEEE Conference on Decision and Control, Linear Algebra Appl., 146, 79-91.

Brockett, R. [1994], The double bracket equation as the solution of a variational problem, in Hamiltonian and gradient flows, algorithms and control, Fields Institute Communications, 3, Amer. Math. Soc., Providence, RI., 69-76.

Chow, B. and D. Knopf [2004], The Ricci Flow: An Introduction, Providence: American Mathematical Society.

Deift, P., T. Nanda, and C. Tomei [1983], Differential equations for the symmetric eigenvalue problem, SIAM J. on Numerical Analysis 20, 1-22.

Deift, P., L. C. Li, T. Nanda and C. Tomei [1992], The Toda flow on a generic orbit is integrable, Mem. Amer. Math. Soc. 100.

Fish, D. [2005], Metriplectic Systems, thesis, Portland State University.

Flaschka, H. [1974], The Toda Lattice, Phys. Rev. B 9, 1924-1925.

Flierl, G. R. and P. J. Morrison [2011], Hamiltonian-Dirac Simulated Annealing: Application to the Calculation of Vortex States, Physica D 240, 212-232 (2011).

Gardner, C. S. [1971], Korteweg-de Vries equation and generalizations. IV. The Korteweg-de Vries equation as a Hamiltonian systems, J. Math. Phys. 12, 1548-1551.

Gay-Balmaz, F. and Holm, D. D. [2012], Parameterizing interaction of disparate scales: Selective decay by Casimir dissipation in fluids, preprint, arXiv:1206.2607v 1.

Grmela, M., Öttinger, H.C. [1997], Dynamics and thermodynamics of complex fluids. I. Development of a general formalism, Phys. Rev. E (3) 56(6), 6620-6632.

Hamilton, R. S. [1982], The inverse function theorem of Nash and Moser, Bull. Amer. Math. Soc. (N.S.) 7(1), 65-222.

Hamilton, R. S. [1982], Three-manifolds with positive Ricci curvature, J. Diff. Geom. 17, 255-306.

Holm, D. D. and J. E. Marsden [1991], The rotor and the pendulum, Symplectic Geometry and Mathematical Physics, P. Donato et. al. eds., Birkhauser 189-203.

Kaufman, A. N. and P. J. Morrison [1982], Algebraic structure of the plasma quasilinear equations, Phys. Lett. A 88, 405-406.

Kaufman, A. N. [1984], Dissipative Hamiltonian systems: a unifying principle, Phys. Lett. A 100, 419-422.

King, F. W. [2009], Hilbert Transforms, two volumes, Encyclopedia of Mathematics and its Applications, 124,125, Cambridge University Press, Cambridge.

Kostant, B. [1979], The solution to a generalized Toda lattice and representation theory, Adv. in Math. 34(3), $195-338$.

Kriegl, A. and P. W. Michor [1997], The Convenient Setting of Global Analysis, Mathematical Surveys and Monographs, 53. American Mathematical Society, Providence, RI.

Liero, M. and A. Mielke [2012], Gradient structures and geodesic convexity for reaction-diffusion systems, preprint.

Marsden, J. E. and T. S. Ratiu [1999], Introduction to Mechanics and Symmetry, Springer-Verlag, Texts in Applied Mathematics, vol. 17; 1994, Second Edition, 1999. 
Medina, A. and Revoy, Ph. [1985], Algèbres de Lie et produit scalaire invariant, Ann. Scient. Ec. Norm Sup., $4^{e}$ série, 18, 553-561.

Mielke, A. [2011], Formulation of thermoelastic dissipative material using GENERIC, Continuum Mech. Thermodyn. 23, 233-256.

Morrison, P. J. [1980], The Maxwell-Vlasov Equations as a Continuous Hamiltonian System, Phys. Lett. A 80, 383386.

Morrison, P. J. [1984a], Bracket formulation for irreversible classical fields, Phys. Lett. A 100, 423-427.

Morrison, P. J. [1984b], Some observations regarding brackets and dissipation, Center for Pure and Applied Mathematics Report PAM228, University of California, Berkeley.

Morrison, P. J. [1986], A paradigm for joined Hamiltonian and dissipative systems, Physica D 18, 410-419.

Morrison, P. J. [1998], Hamiltonian description of the ideal fluid, Rev. Mod. Phys. 70, 467-521.

Morrison, P. J. [2009], Thoughts on brackets and dissipation: old and new, J. Phys: Conf. Ser. 169, 1-12.

Moser, J. [1975], Finitely many mass points on the line under the influence of an exponential potential - an integrable system, in Dynamical Systems, Theory and Applications (Rencontres, Battelle Res. Inst., Seattle, Wash., 1974), pp. 467-497, Lecture Notes in Physics 38, Springer-Verlag, Berlin.

Nambu, Y. [1971], Generalized Hamiltonian dynamics, Phys. Rev. D 7, 2405-2412.

Neeb, K.-H. [2004], Infinite-dimensional Groups and their Representations, in Lie Theory, Progress in Mathematics 228, 213-328. Birkhäuser Boston, Boston,

Neeb, K.-H. [2006], Towards a Lie theory for infinite-dimensional groups, Jap. J. Math. 3rd Series 1(2), 291-468.

Oettinger, H.C. [2006], Beyond Equilibrium Thermodynamics, Wiley.

Ott, E. and R. N. Sudan [1969], Nonlinear theory of ion acoustic waves with Landau damping, Phys. Fluids 12, 23882394.

Otto, F. [2001], The geometry of dissipative evolution equations: the porous medium equation, Com. Partial Diff. Eqs. 26, 101-174.

Palais, R. S. [1968], Foundations of Global Non-Linear Analysis, Benjamin/Cummins Publishing Co., Reading, MA.

Pressley, A. N. [1982], The energy flow on the loop space of a compact Lie group, J. London Math. Soc. (2) 26(3), 557-566.

Pressley, A. and Segal, G. [1986], Loop Groups, Oxford University Press, Oxford.

Ratiu, T. [1980], Involution theorems, in Geometric Methods in Mathematical Physics (Proc. NSF-CBMS Conf., Univ. Lowell, Lowell, Mass., 1979), pp. 219-257, Lecture Notes in Math. 775, Springer-Verlag, Berlin.

Segal, G. [1981], Unitary representations of some infinite dimensional groups, Comm. Math. Phys. 80, 301-342.

J.-M. Souriau [1970], Structure des Systèmes Dynamiques, Dunod, Paris.

Symes, W. W. [1980], Hamiltonian group actions and integrable systems, Physica D 1, 339-374.

Symes, W. W. [1980], Systems of Toda type, inverse spectral problems, and representation theory, Invent. Math. 59(1), $13-51$.

Symes, W. W. [1982], The QR algorithm and scattering for the nonperiodic Toda lattice, Physica D 4, 275-280.

Vallis, G. K., G. Carnevale and W. R. Young [1989], Extremal energy properties and construction of stable solutions of the Euler equations, J. Fluid Mech. 207, 133-152.

Zaharov, V. E. and L. D. Faddeev [1971], The Korteweg-de Vries equation is a fully integrable Hamiltonian system (Russian), Funkcional. Anal. i Priložen. 5(4), 18-27. 